
\title{
28 Research Suare \\ Top 100 Cited Systematic Reviews and Meta-Analyses in The Major Journals of Oral and Maxillofacial Surgery: A Bibliometric Analysis
}

\section{Essam Al-Moraissi ( $\boldsymbol{D}$ dressamalmoraissi@gmail.com )}

Thamar University Faculty of Medicine and Health Sciences: Dhamar University Faculty of Medicine and Health Sciences https://orcid.org/0000-0002-3649-9662

\section{Endi Lanza Galvão}

Universidade Federal dos Vales do Jequitinhonha e Mucuri

\section{Nikolaos Christidis}

Karolinska Institutets folkhälsoakademi: Karolinska Institutet

\section{Saulo Gabriel Falci}

Universidade Federal dos Vales do Jequitinhonha e Mucuri

\section{Research}

Keywords: Bibliometrics, Citation Analysis, Oral and Maxillofacial Surgery, Systemic Reviews, Meta-Analysis

Posted Date: December 17th, 2020

DOI: https://doi.org/10.21203/rs.3.rs-127741/v1

License: (1) This work is licensed under a Creative Commons Attribution 4.0 International License. Read Full License 


\section{Abstract}

Background: The aim of this bibliometric research was to identify and analyze the top 100 cited systematic reviews in the field of oral and maxillofacial surgery.

Methods : Using the Web of Science-database without restrictions on publication year or language, a bibliometric analysis was performed for the five major journals of oral and maxillofacial surgery: International Journal of Oral and Maxillofacial Surgery, Journal of Oral and Maxillofacial Surgery, Journal of Craniomaxillofacial Surgery, British Journal of Oral \& Maxillofacial Surgery and Oral Surgery Oral medicine Oral pathology Oral radiology.

Discussion : The most top-cited systematic review was published in 2015 with a total of 200 citations on survival and success rates of dental implants, consistent with the finding that "pre- and peri-implant surgery and dental implantology", and "craniomaxillofacial deformities and cosmetic surgery" were the most frequently-cited topics (22\% each).

The International Journal of Oral and Maxillofacial Surgery displayed both most citations in total and in average per publication. The outcome of this article can be used as a source of information not just for researchers but also for clinicians and students, and of which areas have a large impact on the field of oral and maxillofacial surgery but cannot reflect the quality of the included systematic reviews.

\section{Systematic review registration: not applicable}

\section{Background}

Decision-making regarding all clinical decisions including choice of treatment is nowadays nationally and internationally based on evidence-based medicine in all fields of medicine such as dentistry and oral and maxillofacial surgery (OMFS) [1, 2]. Randomized controlled trials (RCTs) have been considered the best and strongest study design to answer a specific clinical question, consequently being a guide to the decision making, described as an evidence-based approach. However, in most of the RCTs, a huge sample size is warranted to reach an outcome based on reliable statistics. Performing a RCT with a big sample size is a challenge for any researcher. To solve this problem, a study design called systematic review with metaanalysis was proposed. This type of research allows the researchers to agglutinate data extracted from many RCTs, in only one statistical analysis. Thus, this kind of statistical analysis can provide a more robust and stronger outcome/result about one specific clinical question. Besides that, there are tools to evaluate the quality of RCTs, in order to be able to do recommendations about this specific clinical issue. Therefore, presently systematic reviews and meta-analysis are graded with the highest quality level study design [3].

Another type of research, used to show which impact publications have, is called "bibliometrics". The first citation about bibliometrics was done by Pritchard in 1969 [4]. There are some publications on bibliometric analysis in the field of OMSF, but specified as facial trauma [5], oral cancer [6], and maxillofacial surgery [7]. The citation analysis is a type of bibliometrics which quantifies how many times a publication has been cited after its publication. This information can be efficient to use in order to evaluate which impact a publication has in a specific field, and therefore how important this publication is in the specific field. Thus, the more cited 
the publication is, the greater ability it has to influence clinical decisions [8]. Furthermore, this analysis is an important tool to help the clinicians to identify the best publications in their field of action and to guide them in their decision making based on these publications.

After the millennial shift there was a huge increase in the number of published systematic reviews in medicine and dentistry. Specifically, in the field of OMFS the increase of published systematic reviews was observed after 2010. To our knowledge there are no studies aiming to investigate the most cited systematic reviews in the field of OMFS. Thus, after 10 years of worldwide research it is imperative to know how the systematic reviews are influencing the decision making in the field of OMFS, and to rank the most cited systematic reviews in order to provide a guidance to the clinicians acquisition of knowledge. The aim of this bibliometric research was therefore to identify and analyze the top 100 cited systematic reviews in the field of OMFS.

\section{Methods}

Using the methodology outlined in the standard systematic review methods[9], the authors performed a bibliometric analysis of the top 100 most highly cited systematic reviews in the five major journals of oral and maxillofacial surgery namely: International Journal of Oral and Maxillofacial Surgery (IJOMS), Journal of Oral and Maxillofacial Surgery (JOMS), Journal of Cranio-maxillofacial Surgery (JCMS), British Journal of Oral \& Maxillofacial Surgery (BJOMS) and Oral Surgery Oral medicine Oral pathology Oral radiology (TRIPLEO). A search was performed on 29th October 2020, using the Clarivate Analytics' Web of Science database and there was no restriction on the publication year or the language of the manuscripts. The search strategy was

"systematic review" OR "systematic reviews" OR "meta-analysis" OR "meta-analyses". The results were organized in descending order of citation count.

Every systematic review was stratified into the following categories: dentoalveolar surgery, pre-implant surgery and dental implantology, traumatology, craniomaxillofacial deformities and cosmetic surgery, osteonecrosis of the jaws, pathology, reconstructive surgery, temporomandibular joint (TMJ), basic science research, and emerging technologies

The most cited articles were analyzed regarding the following information: number of citations, publication year, journals, authors, number of authors, methodological design (systematic review or systematic review with meta-analysis), article topic, contributing institution and country. The country of origin and contributing institution of the article was defined by the address provided for the corresponding author. When the paper presented the same citation number the youngest was best ranked.

Number of articles and citations per article were graphed using the Statistical Package for the Social Sciences software (SPSS version 22.0).

\section{Discussion}

The initial search identified 771 articles. The 100 top-cited systematic reviews on the OMFS field are listed by rank order based on the number of citations in Table 1. From a total of 100 systematic reviews only 37 presented meta-analyses. The number of authors ranged between one and 16 (mean $4.12 \pm 2.31$ ). 
These articles have been cited a combined total of 5107 times. The most top-cited article was published in 2015 with a total of 200 citations [10]. Based on the distribution of the 100 articles over the years and their citations per publication, the years 2000 followed by 2006 and 2007 were the most productive years (Figure 1). The earliest systematic review included in this bibliometric analysis was published in 2000 by Lee et al. [11], in JOMS and has been cited 98 times, while the most recently was published in 2018 by Starch-Jensen et al. [12]in the IJOMS and has been cited 33 times. Figure 2 illustrates the distribution of the 100 articles over the years.

The topics "pre- and peri-implant surgery and dental implantology", as well as "craniomaxillofacial deformities and cosmetic surgery" were the most frequently cited topics (22\% each) in the top 1

00 list (Figure 3). The first topic has a total of 1334 citations while the second topic has a total of 1162 citations.

There were 25 different countries of origin and 83 institutions responsible for the highly cited systematic reviews. The leading countries were The Netherlands and Italy with 12 manuscripts each, followed by the USA with 10 articles (Figure 4).

Overall, IJOMS was responsible for 2481 citations, JOMS for 1684, TRIPLEO for 380 citations, JCMFS for 307 , and BJOMS for 255. The mean citation rate per published review followed the same pattern with IJOMS having a mean citation rate of 57.7 citations per review, JOMS 49.5 citations per review, TRIPLEO 47.5 citations per review, JCMFS 38.4 citations per review, and BJOMS 31.9 citations per review. Most of the manuscripts were published in the IJOMS and JOMS (Figure 5).

In the field of (dental)medicine, as in the other fields of science, there is wish reach out, to affect decisions, and to guide the reader in a decision making [8]. Articles reaching more than 100 citations are considered classic, i.e. having a great impact [13]. However, to analyze and understand if the conducted research has any impact or affects decision making one has to analyze how and if the articles do reach out, and which impact they have in their research filed [13]. To do that scientometrics, which is bibliometry in the field of science, is frequently used. This study used citation analysis [14], to identify the publications that have had the greatest impact in the field of OMFS. Although the field of OMFS is very wide, with diverse conditions and treatments, the main finding of this citation analysis indicates that there were only 7 systematic reviews that reached to 100 citations in the field of OMFS, among the included journals. This is in line with previous studies indicating that less $10 \%$ of the published articles reaches up to the status of classic articles [15-17].

Among the classic articles the top cited article was an Italian systematic review on longitudinal studies about the evaluation of survival and success rates of dental implants [10], published in 2015 but already up in 200 citations. Among top-three there was one more systematic review on dental implants also from Italy focusing on different alveolar bone augmentation procedures for implant placement [18], published in 2014 and has now reached 124 citations. It is not surprising that systematic reviews upon dental treatments are top ranked since dental implant surgery is the vast most common surgical procedure next to tooth extractions, in contrast to orthognathic surgery, tumors.[19] 
The second most cited was also a European (from The Netherlands) systematic review on three-dimensional image fusion processes for planning and evaluating orthodontics and orthognathic surgery, published in 2011 having 131 citations. All top-three systematic reviews were published in IJOMS, which is the top journal in the field of OMFS, being ranked in the first quartile (QR1). It has been shown that the journal impact factor answers for $59 \%$ of the variation in the number of citations [20]. Therefore, it is not at surprising finding since most authors are interested in publishing papers in journals with high impact-factors, which also is considered an indication of high quality papers [21,22]. Just outside top-three systematic reviews this citation analysis could show that systematic reviews upon osteoradionecrosis from Hong Kong (112 citations)[23] and Malaysia (100 citations)[24], as well as osteonecrosis from Germany (104 citations)[25]. The same topics were also dominating the rest of the most highly cited systematic reviews.

It has previously been reported that the majority of the top ranked, top cited publications are produced in nations with better economic rankings $[17,26]$. This was also found in this citation analysis indicating that most of the systematic reviews in the field of OMFS are produced in Europe and the US, as well as Hong Kong, with Italy as the most successful country.

One interesting factor is that the top ranked systematic reviews are all published after the year 2010 , however not surprising since only 14 out of the 100 top cited were published in the decade 2000-2010. One common criticism on citation analysis reports is that the outcome is affected by the impact of time [15]. This was, however, not the case in this report on the field of OMFS. In accordance with our results, previous studies have indicated that there are just a few citations the first years, with a peak of citations just before an article-age of 10 years [27].

Another aspect to consider is that only can be used to assess the impact the specific article has on its field by quantifying its recognition, the importance, and also perhaps how common or severe a condition might be, but it cannot reflect the quality of the content in the article [21, 22]. Therefore, it is of great importance to use the outcome of this article as a source of information not just for researchers but also for clinicians and students, and which areas have a large impact on the field of OFMS.

\section{Abbreviations}

OMFS : oral and maxillofacial surgery

TMJ : temporomandibular joint

IJOMS: International Journal of Oral and Maxillofacial Surgery

JOMS: Journal of Oral and Maxillofacial Surgery

JCMS: Journal of Cranio-maxillofacial Surgery

BJOMS: British Journal of Oral \& Maxillofacial Surgery

TRIPLE O: Oral Surgery Oral medicine Oral pathology Oral radiology 


\section{Declarations}

Ethics approval and consent to participate : not applicable

Consent for publication: not applicable

Availability of data and materials: not applicable

Competing interests : The authors declare that they have no competing interests

Funding: None

Authors' contributions: all authors contributed equally in this manuscript. All authors read and approved the final manuscript

Acknowledgements: not applicable

Authors' information (optional)

\section{References}

1. Gogos $\mathrm{C}$, Kodonas K, Fardi A, Economides N: Top 100 cited systematic reviews and meta-analyses in dentistry. Acta Odontol Scand 2020, 78(2):87-97.

2. Beteramia D, Sklavos A, Saha A, Hyam D: A 21-year analysis of the publication patterns and level of scientific evidence in three major oral and maxillofacial surgery journals. Int J Oral Maxillofac Surg 2020.

3. CEBM develops, promotes and disseminates better evidence for healthcare [https://www.cebm.net/category/open-evidence-reviews/]

4. Pritchard A: Statistical bibliography or bibliometrics. Journal of documentation 1969, 25(4):348-349.

5. Tahim A, Patel K, Bridle C, Holmes S: The 100 Most Cited Articles in Facial Trauma: A Bibliometric Analysis. J Oral Maxillofac Surg 2016, 74(11):2240 e2241-2240 e2214.

6. Hassona Y, Qutachi T: A bibliometric analysis of the most cited articles about squamous cell carcinoma of the mouth, lips, and oropharynx. Oral Surg Oral Med Oral Pathol Oral Radiol 2019, 128(1):25-32 e26.

7. Aslam-Pervez N, Lubek JE: Most cited publications in oral and maxillofacial surgery: a bibliometric analysis. Oral Maxillofac Surg 2018, 22(1):25-37.

8. Mogull SA: Accuracy of cited "facts" in medical research articles: A review of study methodology and recalculation of quotation error rate. PLoS One 2017, 12(9):e0184727.

9. Moher D, Shamseer L, Clarke M, Ghersi D, Liberati A, Petticrew M, Shekelle P, Stewart LA, Group P-P:

Preferred reporting items for systematic review and meta-analysis protocols (PRISMA-P) 2015 statement. Systematic Reviews 2015, 4(1):1.

10. Moraschini V, Poubel LA, Ferreira VF, Barboza Edos S: Evaluation of survival and success rates of dental implants reported in longitudinal studies with a follow-up period of at least 10 years: a systematic review. Int J Oral Maxillofac Surg 2015, 44(3):377-388. 
11. Lee JJ, Rouhfar L, Beirne OR: Survival of hydroxyapatite-coated implants: A meta-analytic review. Journal of Oral and Maxillofacial Surgery 2000, 58(12):1372-1379.

12. Starch-Jensen T, Aludden $H$, Hallman M, Dahlin C, Christensen AE, Mordenfeld A: A systematic review and meta-analysis of long-term studies (five or more years) assessing maxillary sinus floor augmentation. International journal of oral and maxillofacial surgery 2018, 47(1):103-116.

13. Heldwein FL, Rhoden EL, Morgentaler A: Classics of urology: a half century history of the most frequently cited articles (1955-2009). Urology 2010, 75(6):1261-1268.

14. Schaer P: Applied informetrics for digital libraries: an overview of foundations, problems and current approaches. Historical Social Research/Historische Sozialforschung 2013:267-281.

15. Sengupta N, Sarode SC, Sarode GS, Gadbail AR, Gondivkar S, Patil S, Patil S: Analysis of 100 most cited articles on forensic odontology. Saudi Dent J 2020, 32(7):321-329.

16. Dmytriw AA, Hui N, Singh T, Nguyen D, Omid-Fard N, Phan K, Kapadia A: Bibliometric evaluation of systematic review and meta analyses published in the top 5 "high-impact" radiology journals. Clin Imaging 2020, 71:52-62.

17. Lai P, Liu YH, Xue JH, He PC, Qiu YQ: The 100 most-cited articles on aortic dissection. BMC CardiovasC Disord 2017, 17(1):30.

18. Milinkovic I, Cordaro L: Are there specific indications for the different alveolar bone augmentation procedures for implant placement? A systematic review. Int J Oral Maxillofac Surg 2014, 43(5):606-625.

19. Elani HW, Starr JR, Da Silva JD, Gallucci GO: Trends in Dental Implant Use in the U.S., 1999-2016, and Projections to 2026. J Dent Res 2018, 97(13):1424-1430.

20. Royle $\mathrm{P}$, Kandala NB, Barnard K, Waugh N: Bibliometrics of systematic reviews: analysis of citation rates and journal impact factors. Syst Rev 2013, 2:74.

21. Gondivkar SM, Sarode SC, Gadbail AR, Gondivkar RS, Choudhary N, Patil S: Citation Classics in Cone Beam Computed Tomography: The 100 Top-Cited Articles. Int J Dent 2018, 2018:9423281.

22. Gondivkar SM, Sarode SC, Gadbail AR, Gondivkar RS, Chole R, Sarode GS: Bibliometric analysis of 100 most cited articles on oral submucous fibrosis. J Oral Pathol Med 2018, 47(8):781-787.

23. Nabil S, Samman N: Incidence and prevention of osteoradionecrosis after dental extraction in irradiated patients: a systematic review. Int J Oral Maxillofac Surg 2011, 40(3):229-243.

24. Nabil S, Samman N: Risk factors for osteoradionecrosis after head and neck radiation: a systematic review. Oral Surg Oral Med Oral Pathol Oral Radiol 2012, 113(1):54-69.

25. Fliefel R, Tröltzsch M, Kühnisch J, Ehrenfeld M, Otto S: Treatment strategies and outcomes of bisphosphonate-related osteonecrosis of the jaw (BRONJ) with characterization of patients: a systematic review. Int J Oral Maxillofac Surg 2015, 44(5):568-585.

26. Pena-Cristobal M, Diniz-Freitas M, Monteiro L, Diz Dios P, Warnakulasuriya S: The 100 most cited articles on oral cancer. J Oral Pathol Med 2018, 47(4):333-344.

27. Eom YH, Fortunato S: Characterizing and modeling citation dynamics. PLoS One 2011, 6(9):e24926.

\section{Tables}


Table 1. The top 100 cited systematic review and meta-analyses in oral and maxillofacial surgery. 


\begin{tabular}{|c|c|c|c|c|c|c|}
\hline Rank & Author & Title & $\begin{array}{l}\text { Publication } \\
\text { Year }\end{array}$ & Journal & Institution & Citations \\
\hline 1 & $\begin{array}{l}\text { Moraschini } \\
\text { V et al. }\end{array}$ & $\begin{array}{l}\text { Evaluation of } \\
\text { survival and } \\
\text { success rates of } \\
\text { dental implants } \\
\text { reported in } \\
\text { longitudinal studies } \\
\text { with a follow-up } \\
\text { period of at least } 10 \\
\text { years: a systematic } \\
\text { review }\end{array}$ & 2015 & IJMOS & $\begin{array}{l}\text { Fluminense } \\
\text { Federal University }\end{array}$ & 200 \\
\hline 2 & $\begin{array}{l}\text { Plooij JM et } \\
\text { al. }\end{array}$ & $\begin{array}{l}\text { Digital three- } \\
\text { dimensional image } \\
\text { fusion processes for } \\
\text { planning and } \\
\text { evaluating } \\
\text { orthodontics and } \\
\text { orthognathic } \\
\text { surgery. A } \\
\text { systematic review }\end{array}$ & 2011 & IJMOS & $\begin{array}{l}\text { 3D Facial } \\
\text { Imaging Research } \\
\text { Group Nijmegen- } \\
\text { Bruges }\end{array}$ & 131 \\
\hline 3 & $\begin{array}{l}\text { Milinkovic I } \\
\text { et al. }\end{array}$ & $\begin{array}{l}\text { Are there specific } \\
\text { indications for the } \\
\text { different alveolar } \\
\text { bone augmentation } \\
\text { procedures for } \\
\text { implant placement? } \\
\text { A systematic review }\end{array}$ & 2014 & IJMOS & $\begin{array}{l}\text { Eastman Dental } \\
\text { Hospital }\end{array}$ & 124 \\
\hline 4 & $\begin{array}{l}\text { Milinkovic I, } \\
\text { Cordaro L }\end{array}$ & $\begin{array}{l}\text { Incidence and } \\
\text { prevention of } \\
\text { osteoradionecrosis } \\
\text { after dental } \\
\text { extraction in } \\
\text { irradiated patients: } \\
\text { a systematic review }\end{array}$ & 2011 & IJMOS & $\begin{array}{l}\text { University of } \\
\text { Hong Kong }\end{array}$ & 112 \\
\hline 5 & $\begin{array}{l}\text { Nabil S, } \\
\text { Samman N }\end{array}$ & $\begin{array}{l}\text { Stability After } \\
\text { Bilateral Sagittal } \\
\text { Split Osteotomy } \\
\text { Advancement } \\
\text { Surgery With Rigid } \\
\text { Internal Fixation: A } \\
\text { Systematic Review }\end{array}$ & 2009 & JOMS & $\begin{array}{l}\text { University of } \\
\text { Geneva }\end{array}$ & 107 \\
\hline 6 & $\begin{array}{l}\text { Joss CU, } \\
\text { Vassalli IM }\end{array}$ & $\begin{array}{l}\text { Treatment } \\
\text { strategies and } \\
\text { outcomes of } \\
\text { bisphosphonate- } \\
\text { related } \\
\text { osteonecrosis of the } \\
\text { jaw (BRONJ) with } \\
\text { characterization of } \\
\text { patients: a } \\
\text { systematic review }\end{array}$ & 2015 & IJMOS & Universiat Munich & 104 \\
\hline 7 & $\begin{array}{l}\text { Nabil S, } \\
\text { Samman N }\end{array}$ & $\begin{array}{l}\text { Risk factors for } \\
\text { osteoradionecrosis } \\
\text { after head and neck }\end{array}$ & 2012 & TRIPLEO & $\begin{array}{l}\text { National } \\
\text { University of } \\
\text { Malaysia }\end{array}$ & 100 \\
\hline
\end{tabular}


radiation: a

systematic review

\begin{tabular}{|c|c|c|c|c|c|c|}
\hline 8 & Lee JJ et al. & $\begin{array}{l}\text { Survival of } \\
\text { hydroxyapatite- } \\
\text { coated implants: A } \\
\text { meta-analytic } \\
\text { review }\end{array}$ & 2000 & JOMS & $\begin{array}{l}\text { University of } \\
\text { Washington }\end{array}$ & 98 \\
\hline 9 & $\begin{array}{l}\text { Cheung LK, } \\
\text { Chua HDP }\end{array}$ & $\begin{array}{l}\text { A meta-analysis of } \\
\text { cleft maxillary } \\
\text { osteotomy and } \\
\text { distraction } \\
\text { osteogenesis }\end{array}$ & 2006 & IJMOS & $\begin{array}{l}\text { The University of } \\
\text { Hong Kong }\end{array}$ & 97 \\
\hline 10 & $\begin{array}{l}\text { Lau SL, } \\
\text { Samman N }\end{array}$ & $\begin{array}{l}\text { Recurrence related } \\
\text { to treatment } \\
\text { modalities of } \\
\text { unicystic } \\
\text { ameloblastoma: a } \\
\text { systematic review }\end{array}$ & 2006 & IJMOS & $\begin{array}{l}\text { The University of } \\
\text { Hong Kong }\end{array}$ & 96 \\
\hline 11 & $\begin{array}{l}\text { Colella G et } \\
\text { al. }\end{array}$ & $\begin{array}{l}\text { Fine-Needle } \\
\text { Aspiration Cytology } \\
\text { of Salivary Gland } \\
\text { Lesions: A } \\
\text { Systematic Review }\end{array}$ & 2010 & JOMS & $\begin{array}{l}\text { Second University } \\
\text { of Naples }\end{array}$ & 91 \\
\hline 12 & $\begin{array}{l}\text { Clementini } \\
\text { M et al. }\end{array}$ & $\begin{array}{l}\text { Success rate of } \\
\text { dental implants } \\
\text { inserted in } \\
\text { horizontal and } \\
\text { vertical guided bone } \\
\text { regenerated areas: a } \\
\text { systematic review }\end{array}$ & 2012 & IJMOS & $\begin{array}{l}\text { University Tor } \\
\text { Vergata }\end{array}$ & 90 \\
\hline 13 & $\begin{array}{l}\text { Ren YF, } \\
\text { Malmstrom } \\
\text { HS }\end{array}$ & $\begin{array}{l}\text { Effectiveness of } \\
\text { antibiotic } \\
\text { prophylaxis in third } \\
\text { molar surgery: A } \\
\text { meta-analysis of } \\
\text { randomized } \\
\text { controlled clinical } \\
\text { trials }\end{array}$ & 2007 & JOMS & $\begin{array}{l}\text { University of } \\
\text { Rochester }\end{array}$ & 89 \\
\hline 14 & $\begin{array}{l}\text { Stokbro K et } \\
\text { al. }\end{array}$ & $\begin{array}{l}\text { Virtual planning in } \\
\text { orthognathic } \\
\text { surgery }\end{array}$ & 2014 & IJMOS & $\begin{array}{l}\text { Odense University } \\
\text { Hospital }\end{array}$ & 88 \\
\hline 15 & $\begin{array}{l}\text { Rickert D et } \\
\text { al. }\end{array}$ & $\begin{array}{l}\text { Maxillary sinus lift } \\
\text { with solely } \\
\text { autogenous bone } \\
\text { compared to a } \\
\text { combination of } \\
\text { autogenous bone } \\
\text { and growth factors } \\
\text { or (solely) bone } \\
\text { substitutes. A } \\
\text { systematic review }\end{array}$ & 2012 & IJMOS & $\begin{array}{l}\text { University } \\
\text { Medical Center } \\
\text { Groningen }\end{array}$ & 78 \\
\hline 16 & $\begin{array}{l}\text { Goiato MC } \\
\text { et al. }\end{array}$ & $\begin{array}{l}\text { Longevity of dental } \\
\text { implants in type IV }\end{array}$ & 2014 & IJMOS & $\begin{array}{l}\text { Universidade } \\
\text { Estadual Paulista }\end{array}$ & 76 \\
\hline
\end{tabular}

Page 10/40 


\begin{tabular}{|c|c|c|c|c|c|c|}
\hline & & $\begin{array}{l}\text { bone: a systematic } \\
\text { review }\end{array}$ & & & & \\
\hline 17 & $\begin{array}{l}\text { Markiewicz } \\
\text { MR et al. }\end{array}$ & $\begin{array}{l}\text { Corticosteroids } \\
\text { reduce } \\
\text { postoperative } \\
\text { morbidity after third } \\
\text { molar surgery: A } \\
\text { systematic review } \\
\text { and meta-analysis }\end{array}$ & 2008 & JOMS & $\begin{array}{l}\text { Oregon Health } \\
\text { and Science } \\
\text { University }\end{array}$ & 76 \\
\hline 18 & $\begin{array}{l}\text { Merkx MAW } \\
\text { et al. }\end{array}$ & $\begin{array}{l}\text { Assessment of the } \\
\text { value of anorganic } \\
\text { bone additives in } \\
\text { sinus floor } \\
\text { augmentation: a } \\
\text { review of clinical } \\
\text { reports }\end{array}$ & 2003 & IJMOS & $\begin{array}{l}\text { University of } \\
\text { Nijmegen }\end{array}$ & 71 \\
\hline 19 & $\begin{array}{l}\text { Colella G et } \\
\text { al. }\end{array}$ & $\begin{array}{l}\text { Neurosensory } \\
\text { disturbance of the } \\
\text { inferior alveolar } \\
\text { nerve after bilateral } \\
\text { sagittal split } \\
\text { osteotomy: A } \\
\text { systematic review }\end{array}$ & 2007 & JOMS & $\begin{array}{l}\text { Second University } \\
\text { of Naples }\end{array}$ & 68 \\
\hline 20 & $\begin{array}{l}\text { Soh CL, } \\
\text { Narayanan } \\
\text { V }\end{array}$ & $\begin{array}{l}\text { Quality of life } \\
\text { assessment in } \\
\text { patients with } \\
\text { dentofacial } \\
\text { deformity } \\
\text { undergoing } \\
\text { orthognathic } \\
\text { surgery-A } \\
\text { systematic review }\end{array}$ & 2013 & IJMOS & $\begin{array}{l}\text { Saveetha } \\
\text { University, } \\
\text { Vellappanchavadi }\end{array}$ & 63 \\
\hline 21 & $\begin{array}{l}\text { Clementini } \\
\text { M et al. }\end{array}$ & $\begin{array}{l}\text { Systemic risk } \\
\text { factors for peri- } \\
\text { implant bone loss: a } \\
\text { systematic review } \\
\text { and meta-analysis }\end{array}$ & 2014 & IJMOS & $\begin{array}{l}\text { Independent } \\
\text { Researcher }\end{array}$ & 60 \\
\hline 22 & $\begin{array}{l}\text { Kaczmarzyk } \\
\text { T et al. }\end{array}$ & $\begin{array}{l}\text { A systematic review } \\
\text { of the recurrence } \\
\text { rate for keratocystic } \\
\text { odontogenic tumour } \\
\text { in relation to } \\
\text { treatment } \\
\text { modalities }\end{array}$ & 2012 & IJMOS & $\begin{array}{l}\text { Jagiellonian } \\
\text { University } \\
\text { Medical College }\end{array}$ & 59 \\
\hline 23 & $\begin{array}{l}\text { Andreasen } \\
\text { JO et al. }\end{array}$ & $\begin{array}{l}\text { A systematic review } \\
\text { of prophylactic } \\
\text { antibiotics in the } \\
\text { surgical treatment } \\
\text { of maxillofacial } \\
\text { fractures }\end{array}$ & 2006 & JOMS & $\begin{array}{l}\text { University } \\
\text { Hospital } \\
\text { (Rigshospitalet) }\end{array}$ & 59 \\
\hline 24 & $\begin{array}{l}\text { Gunarajah } \\
\text { DR, } \\
\text { Samman N }\end{array}$ & $\begin{array}{l}\text { Biomaterials for } \\
\text { Repair of Orbital } \\
\text { Floor Blowout }\end{array}$ & 2013 & JOMS & $\begin{array}{l}\text { University of } \\
\text { Hong Kong }\end{array}$ & 57 \\
\hline
\end{tabular}


Fractures: A

Systematic Review

\begin{tabular}{|c|c|c|c|c|c|c|}
\hline 25 & $\begin{array}{l}\text { Kaipatur NR, } \\
\text { Flores-Mir C }\end{array}$ & $\begin{array}{l}\text { Accuracy of } \\
\text { Computer Programs } \\
\text { in Predicting } \\
\text { Orthognathic } \\
\text { Surgery Soft Tissue } \\
\text { Response }\end{array}$ & 2009 & JOMS & $\begin{array}{l}\text { University of } \\
\text { Alberta }\end{array}$ & 56 \\
\hline 26 & $\begin{array}{l}\text { Dan AEB et } \\
\text { al. }\end{array}$ & $\begin{array}{l}\text { Corticosteroid } \\
\text { Administration in } \\
\text { Oral and } \\
\text { Orthognathic } \\
\text { Surgery: A } \\
\text { Systematic Review } \\
\text { of the Literature and } \\
\text { Meta-Analysis }\end{array}$ & 2010 & JOMS & $\begin{array}{l}\text { University of } \\
\text { Copenhagen }\end{array}$ & 54 \\
\hline 27 & $\begin{array}{l}\text { Al-Moraissi } \\
\text { EA, Ellis, E }\end{array}$ & $\begin{array}{l}\text { Surgical Treatment } \\
\text { of Adult Mandibular } \\
\text { Condylar Fractures } \\
\text { Provides Better } \\
\text { Outcomes Than } \\
\text { Closed Treatment: A } \\
\text { Systematic Review } \\
\text { and Meta-Analysis }\end{array}$ & 2015 & JOMS & $\begin{array}{l}\text { Thamar } \\
\text { University }\end{array}$ & 53 \\
\hline 28 & $\begin{array}{l}\text { Mattos CT } \\
\text { et al. }\end{array}$ & $\begin{array}{l}\text { Effects of } \\
\text { orthognathic } \\
\text { surgery on } \\
\text { oropharyngeal } \\
\text { airway: a meta- } \\
\text { analysis }\end{array}$ & 2011 & IJMOS & $\begin{array}{l}\text { Federal University } \\
\text { of Rio de Janeiro }\end{array}$ & 53 \\
\hline 29 & $\begin{array}{l}\text { Mohlhenrich } \\
\text { SC et al. }\end{array}$ & $\begin{array}{l}\text { Heat generation and } \\
\text { drill wear during } \\
\text { dental implant site } \\
\text { preparation: } \\
\text { systematic review }\end{array}$ & 2015 & BJOMS & $\begin{array}{l}\text { Aachen University } \\
\text { Hospital }\end{array}$ & 51 \\
\hline 30 & $\begin{array}{l}\text { Boffano P et } \\
\text { al. }\end{array}$ & $\begin{array}{l}\text { Aetiology of } \\
\text { maxillofacial } \\
\text { fractures: a review } \\
\text { of published studies } \\
\text { during the last } 30 \\
\text { years }\end{array}$ & 2014 & BJOMS & $\begin{array}{l}\text { University of } \\
\text { Amsterdan }\end{array}$ & 51 \\
\hline 31 & $\begin{array}{l}\text { Van } \\
\text { Diermen DE } \\
\text { et al. }\end{array}$ & $\begin{array}{l}\text { Management } \\
\text { recommendations } \\
\text { for invasive dental } \\
\text { treatment in } \\
\text { patients using oral } \\
\text { antithrombotic } \\
\text { medication, } \\
\text { including novel oral } \\
\text { anticoagulants }\end{array}$ & 2013 & TRIPLEO & $\begin{array}{l}\text { Academic Centre } \\
\text { for Dentistry } \\
\text { Amsterdam }\end{array}$ & 51 \\
\hline 32 & $\begin{array}{l}\text { Reston JT, } \\
\text { Turkelson } \\
\text { CM }\end{array}$ & $\begin{array}{l}\text { Meta-analysis of } \\
\text { surgical treatments } \\
\text { for }\end{array}$ & 2003 & JOMS & $\begin{array}{l}\text { Department of } \\
\text { Health } \\
\text { Technology } \\
\text { Assessment, ECRI }\end{array}$ & 51 \\
\hline
\end{tabular}

Page $12 / 40$ 


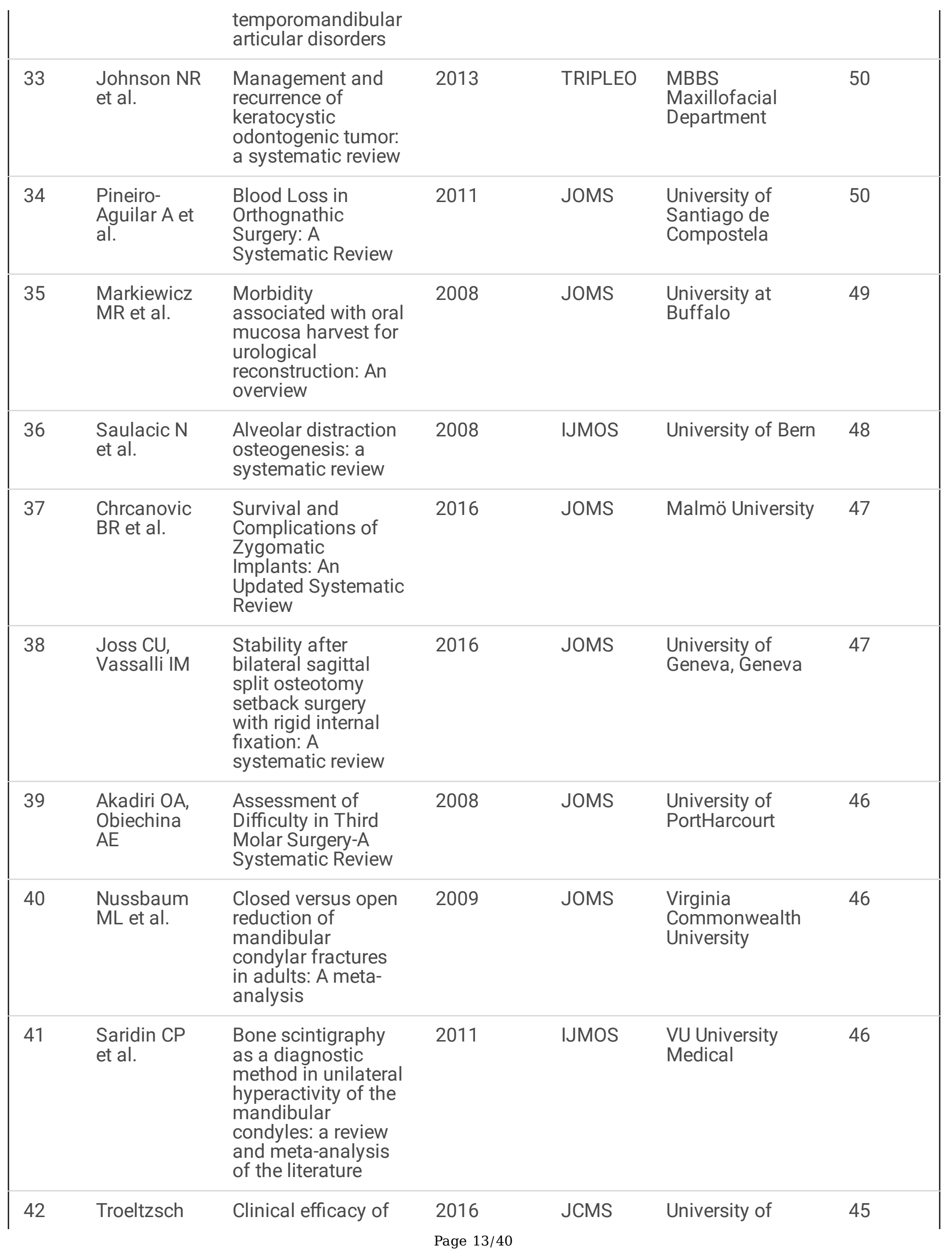




\begin{tabular}{|c|c|c|c|c|c|c|}
\hline & M et al. & $\begin{array}{l}\text { grafting materials in } \\
\text { alveolar ridge } \\
\text { augmentation: A } \\
\text { systematic } \\
\text { reviewClinical } \\
\text { efficacy of grafting } \\
\text { materials in alveolar } \\
\text { ridge augmentation: } \\
\text { A systematic review }\end{array}$ & & & Goettingen & \\
\hline 43 & $\begin{array}{l}\text { Kyzas PA et } \\
\text { al. }\end{array}$ & $\begin{array}{l}\text { The treatment of } \\
\text { mandibular condyle } \\
\text { fractures: A meta- } \\
\text { analysis }\end{array}$ & 2012 & JCMS & $\begin{array}{l}\text { North Manchester } \\
\text { General Hospital }\end{array}$ & 45 \\
\hline 44 & $\begin{array}{l}\text { Del Fabbro } \\
\text { M et al. }\end{array}$ & $\begin{array}{l}\text { Is autologous } \\
\text { platelet concentrate } \\
\text { beneficial for post- } \\
\text { extraction socket } \\
\text { healing? A } \\
\text { systematic review }\end{array}$ & 2011 & IJMOS & $\begin{array}{l}\text { University of } \\
\text { Milan }\end{array}$ & 45 \\
\hline 45 & $\begin{array}{l}\text { Stableforth } \\
\text { WD et al. }\end{array}$ & $\begin{array}{l}\text { A systematic review } \\
\text { of the role of } \\
\text { immunonutrition in } \\
\text { patients undergoing } \\
\text { surgery for head } \\
\text { and neck cancer }\end{array}$ & 2009 & IJMOS & Derriford Hospital & 45 \\
\hline 46 & $\begin{array}{l}\text { Khojasteh A } \\
\text { et al. }\end{array}$ & $\begin{array}{l}\text { Effects of different } \\
\text { growth factors and } \\
\text { carriers on bone } \\
\text { regeneration: a } \\
\text { systematic review }\end{array}$ & 2013 & TRIPLEO & $\begin{array}{l}\text { Shahid Beheshti } \\
\text { University of } \\
\text { Medical Sciences }\end{array}$ & 44 \\
\hline 47 & $\begin{array}{l}\text { Jensen T et } \\
\text { al. }\end{array}$ & $\begin{array}{l}\text { Maxillary sinus floor } \\
\text { augmentation with } \\
\text { Bio-Oss or Bio-Oss } \\
\text { mixed with } \\
\text { autogenous bone as } \\
\text { graft in animals: a } \\
\text { systematic review }\end{array}$ & 2012 & IJMOS & $\begin{array}{l}\text { Aarhus University } \\
\text { Hospital }\end{array}$ & 44 \\
\hline 48 & $\begin{array}{l}\text { Verstraaten } \\
\mathrm{J} \text { et al. }\end{array}$ & $\begin{array}{l}\text { A systematic review } \\
\text { of the effects of } \\
\text { bone-borne surgical } \\
\text { assisted rapid } \\
\text { maxillary expansion }\end{array}$ & 2010 & JCMS & $\begin{array}{l}\text { Radboud } \\
\text { University } \\
\text { Nijmegen Medical } \\
\text { Centre }\end{array}$ & 44 \\
\hline 49 & Atieh MA & $\begin{array}{l}\text { Diagnostic } \\
\text { Accuracy of } \\
\text { Panoramic } \\
\text { Radiography in } \\
\text { Determining } \\
\text { Relationship } \\
\text { Between Inferior } \\
\text { Alveolar Nerve and } \\
\text { Mandibular Third } \\
\text { Molar }\end{array}$ & 2010 & JOMS & $\begin{array}{l}\text { University of } \\
\text { Otago }\end{array}$ & 43 \\
\hline 50 & $\begin{array}{l}\text { Dubois L et } \\
\text { al. }\end{array}$ & $\begin{array}{l}\text { Controversies in } \\
\text { orbital }\end{array}$ & 2015 & IJMOS & $\begin{array}{l}\text { University of } \\
\text { Amsterdam }\end{array}$ & 42 \\
\hline
\end{tabular}




\begin{tabular}{|c|c|c|c|c|c|c|}
\hline & & $\begin{array}{l}\text { reconstruction-I. } \\
\text { Defect-driven orbital } \\
\text { reconstruction: A } \\
\text { systematic review }\end{array}$ & & & & \\
\hline 51 & $\begin{array}{l}\text { Agostini T et } \\
\text { al. }\end{array}$ & $\begin{array}{l}\text { Anterolateral thigh } \\
\text { flap: Systematic } \\
\text { literature review of } \\
\text { specific donor-site } \\
\text { complications and } \\
\text { their management }\end{array}$ & 2013 & JCMS & $\begin{array}{l}\text { CTO-AOUC, } \\
\text { Department of } \\
\text { Traumatology } \\
\text { and Maxillofacial } \\
\text { Surger }\end{array}$ & 42 \\
\hline 52 & $\begin{array}{l}\text { Pirklbauer K } \\
\text { et al. }\end{array}$ & $\begin{array}{l}\text { Maxillomandibular } \\
\text { Advancement for } \\
\text { Treatment of } \\
\text { Obstructive Sleep } \\
\text { Apnea Syndrome: A } \\
\text { Systematic Review }\end{array}$ & 2011 & JOMS & $\begin{array}{l}\text { Medical } \\
\text { University of } \\
\text { Vienna }\end{array}$ & 41 \\
\hline 53 & $\begin{array}{l}\text { Moraschini } \\
\text { V, Barboza } \\
\text { EDP }\end{array}$ & $\begin{array}{l}\text { Success of dental } \\
\text { implants in smokers } \\
\text { and non-smokers: a } \\
\text { systematic review } \\
\text { and meta-analysis }\end{array}$ & 2016 & IJMOS & $\begin{array}{l}\text { Fluminense } \\
\text { Federal University }\end{array}$ & 41 \\
\hline 54 & $\begin{array}{l}\text { Foresta E et } \\
\text { al. }\end{array}$ & $\begin{array}{l}\text { Pleomorphic } \\
\text { adenoma and } \\
\text { benign parotid } \\
\text { tumors: } \\
\text { extracapsular } \\
\text { dissection vs } \\
\text { superficial } \\
\text { parotidectomy- } \\
\text { review of literature } \\
\text { and meta-analysis }\end{array}$ & 2014 & TRIPLEO & $\begin{array}{l}\text { Catholic } \\
\text { University } \\
\text { Medical School }\end{array}$ & 41 \\
\hline 55 & $\begin{array}{l}\text { Pluijmers et } \\
\text { al. }\end{array}$ & $\begin{array}{l}\text { Mandibular } \\
\text { reconstruction in the } \\
\text { growing patient with } \\
\text { unilateral } \\
\text { craniofacial } \\
\text { microsomia: a } \\
\text { systematic review }\end{array}$ & 2014 & IJMOS & $\begin{array}{l}\text { Erasmus } \\
\text { University } \\
\text { Medical Center }\end{array}$ & 40 \\
\hline 56 & $\begin{array}{l}\text { Guarda- } \\
\text { Nardini L et } \\
\text { al. }\end{array}$ & $\begin{array}{l}\text { Synovial } \\
\text { chondromatosis of } \\
\text { the } \\
\text { temporomandibular } \\
\text { joint: a case } \\
\text { description with } \\
\text { systematic literature } \\
\text { review }\end{array}$ & 2010 & IJMOS & $\begin{array}{l}\text { University of } \\
\text { Padova }\end{array}$ & 40 \\
\hline 57 & $\begin{array}{l}\text { Kelly MP et } \\
\text { al. }\end{array}$ & $\begin{array}{l}\text { Systematic Review } \\
\text { and Meta-Analysis } \\
\text { of Recombinant } \\
\text { Human Bone } \\
\text { Morphogenetic } \\
\text { Protein-2 in } \\
\text { Localized Alveolar } \\
\text { Ridge and Maxillary } \\
\text { Sinus Augmentation }\end{array}$ & 2016 & JOMS & $\begin{array}{l}\text { University of } \\
\text { Wisconsin School } \\
\text { of Medicine and } \\
\text { Public Health }\end{array}$ & 39 \\
\hline
\end{tabular}




\begin{tabular}{|c|c|c|c|c|c|c|}
\hline 58 & $\begin{array}{l}\text { Brignardello- } \\
\text { Petersen } \mathrm{R} \\
\text { et al. }\end{array}$ & $\begin{array}{l}\text { Is Adjuvant Laser } \\
\text { Therapy Effective } \\
\text { for Preventing Pain, } \\
\text { Swelling, and } \\
\text { Trismus After } \\
\text { Surgical Removal of } \\
\text { Impacted } \\
\text { Mandibular Third } \\
\text { Molars? A } \\
\text { Systematic Review } \\
\text { and Meta-Analysis }\end{array}$ & 2012 & JOMS & $\begin{array}{l}\text { University of } \\
\text { Toronto, Toronto }\end{array}$ & 39 \\
\hline 59 & $\begin{array}{l}\text { Dubois L et } \\
\text { al. }\end{array}$ & $\begin{array}{l}\text { Controversies in } \\
\text { orbital } \\
\text { reconstruction-II. } \\
\text { Timing of post- } \\
\text { traumatic orbital } \\
\text { reconstruction: A } \\
\text { systematic review }\end{array}$ & 2015 & IJMOS & $\begin{array}{l}\text { University of } \\
\text { Amsterdam }\end{array}$ & 39 \\
\hline 60 & $\begin{array}{l}\text { Goiato MC } \\
\text { et al. }\end{array}$ & $\begin{array}{l}\text { Implants in the } \\
\text { zygomatic bone for } \\
\text { maxillary prosthetic } \\
\text { rehabilitation: a } \\
\text { systematic review }\end{array}$ & 2014 & IJMOS & $\begin{array}{l}\text { Araçatuba Dental } \\
\text { School UNESP }\end{array}$ & 39 \\
\hline 61 & $\begin{array}{l}\text { Ata-Ali J et } \\
\text { al. }\end{array}$ & $\begin{array}{l}\text { Do antibiotics } \\
\text { decrease implant } \\
\text { failure and } \\
\text { postoperative } \\
\text { infections? A } \\
\text { systematic review } \\
\text { and meta-analysis }\end{array}$ & 2014 & IJMOS & $\begin{array}{l}\text { Valencia } \\
\text { University }\end{array}$ & 39 \\
\hline 62 & $\begin{array}{l}\text { Brown JS et } \\
\text { al. }\end{array}$ & $\begin{array}{l}\text { Systematic review } \\
\text { of the current } \\
\text { evidence in the use } \\
\text { of postoperative } \\
\text { radiotherapy for oral } \\
\text { squamous cell } \\
\text { carcinoma }\end{array}$ & 2012 & BJOMS & $\begin{array}{l}\text { University } \\
\text { Hospital } \\
\text { AintreeLowerLane }\end{array}$ & 39 \\
\hline 63 & Klug $C$ et al. & $\begin{array}{l}\text { Preoperative } \\
\text { chemoradiotherapy } \\
\text { in the management } \\
\text { of oral cancer: A } \\
\text { review }\end{array}$ & 2008 & JCMS & $\begin{array}{l}\text { Medical } \\
\text { University of } \\
\text { Vienna }\end{array}$ & 39 \\
\hline 64 & $\begin{array}{l}\text { Katsnelson } \\
\text { A et al. }\end{array}$ & $\begin{array}{l}\text { Operative } \\
\text { Management of } \\
\text { Temporomandibular } \\
\text { Joint Ankylosis: A } \\
\text { Systematic Review } \\
\text { and Meta-Analysis }\end{array}$ & 2012 & JOMS & $\begin{array}{l}\text { Harvard School } \\
\text { of Dental } \\
\text { Medicine }\end{array}$ & 36 \\
\hline 65 & Kyzas PA & $\begin{array}{l}\text { Use of Antibiotics in } \\
\text { the Treatment of } \\
\text { Mandible Fractures: } \\
\text { A Systematic } \\
\text { Review }\end{array}$ & 2011 & JOMS & $\begin{array}{l}\text { Blackburn Royal } \\
\text { Infirmar }\end{array}$ & 36 \\
\hline 66 & Patterson & Corticotomies and & $\begin{array}{l}2016 \\
\text { Page 16/40 }\end{array}$ & JOMS & University of & 35 \\
\hline
\end{tabular}




\begin{tabular}{|c|c|c|c|c|c|c|}
\hline & BM et al. & $\begin{array}{l}\text { Orthodontic Tooth } \\
\text { Movement: A } \\
\text { Systematic Review }\end{array}$ & & & Sydney & \\
\hline 67 & $\begin{array}{l}\text { Moraschini } \\
\text { V et al. }\end{array}$ & $\begin{array}{l}\text { Implant survival } \\
\text { rates, marginal bone } \\
\text { level changes, and } \\
\text { complications in } \\
\text { full-mouth } \\
\text { rehabilitation with } \\
\text { flapless computer- } \\
\text { guided surgery: a } \\
\text { systematic review } \\
\text { and meta-analysis }\end{array}$ & 2015 & IJMOS & $\begin{array}{l}\text { Fluminense } \\
\text { Federal University }\end{array}$ & 35 \\
\hline 68 & $\begin{array}{l}\text { Chrcanovic } \\
\text { BR et al. }\end{array}$ & $\begin{array}{l}\text { Immediately loaded } \\
\text { non-submerged } \\
\text { versus delayed } \\
\text { loaded submerged } \\
\text { dental implants: A } \\
\text { meta-analysis }\end{array}$ & 2015 & IJMOS & Malmö University & 35 \\
\hline 69 & $\begin{array}{l}\text { Tsui WK et } \\
\text { al. }\end{array}$ & $\begin{array}{l}\text { Bone anchor } \\
\text { systems for } \\
\text { orthodontic } \\
\text { application: a } \\
\text { systematic review }\end{array}$ & 2012 & IJMOS & $\begin{array}{l}\text { University of } \\
\text { Hong Kong }\end{array}$ & 35 \\
\hline 70 & $\begin{array}{l}\text { Azarmehr I } \\
\text { et al. }\end{array}$ & $\begin{array}{l}\text { Surgical Navigation: } \\
\text { A Systematic } \\
\text { Review of } \\
\text { Indications, } \\
\text { Treatments, and } \\
\text { Outcomes in Oral } \\
\text { and Maxillofacial } \\
\text { Surgery }\end{array}$ & 2017 & JOMS & $\begin{array}{l}\text { Odense University } \\
\text { Hospital }\end{array}$ & 34 \\
\hline 71 & Aliko A et al. & $\begin{array}{l}\text { World Workshop on } \\
\text { Oral Medicine Vl: } \\
\text { clinical implications } \\
\text { of medication- } \\
\text { induced salivary } \\
\text { gland dysfunction }\end{array}$ & 2015 & TRIPLEO & $\begin{array}{l}\text { Tel-Aviv Sourasky } \\
\text { Medical Center } \\
\text { and Saliwell }\end{array}$ & 34 \\
\hline 72 & $\begin{array}{l}\text { Raijmakers } \\
\text { PG et al. }\end{array}$ & $\begin{array}{l}\text { Female } \\
\text { Predominance and } \\
\text { Effect of Gender on } \\
\text { Unilateral Condylar } \\
\text { Hyperplasia: A } \\
\text { Review and Meta- } \\
\text { Analysis }\end{array}$ & 2012 & JOMS & $\begin{array}{l}\text { VU University } \\
\text { Medical Centre, } \\
\text { de Boelelaan }\end{array}$ & 33 \\
\hline 73 & $\begin{array}{l}\text { Rachidi S; et } \\
\text { al. }\end{array}$ & $\begin{array}{l}\text { Melanotic } \\
\text { Neuroectodermal } \\
\text { Tumor of Infancy: A } \\
\text { Systematic Review }\end{array}$ & 2015 & JOMS & $\begin{array}{l}\text { Medical } \\
\text { University of } \\
\text { South Carolina }\end{array}$ & 33 \\
\hline 74 & $\begin{array}{l}\text { Starch- } \\
\text { Jensen T; et } \\
\text { al. }\end{array}$ & $\begin{array}{l}\text { A systematic review } \\
\text { and meta-analysis } \\
\text { of long-term studies } \\
\text { (five or more years) } \\
\text { assessing maxillary }\end{array}$ & 2018 & IJMOS & $\begin{array}{l}\text { Aalborg } \\
\text { University } \\
\text { Hospital }\end{array}$ & 33 \\
\hline
\end{tabular}




\begin{tabular}{|c|c|c|c|c|c|c|}
\hline & & $\begin{array}{l}\text { sinus floor } \\
\text { augmentation }\end{array}$ & & & & \\
\hline 75 & $\begin{array}{l}\text { Pocaterra A. } \\
\text { et al. }\end{array}$ & $\begin{array}{l}\text { Effectiveness of } \\
\text { platelet-rich plasma } \\
\text { as an adjunctive } \\
\text { material to bone } \\
\text { graft: a systematic } \\
\text { review and meta- } \\
\text { analysis of } \\
\text { randomized } \\
\text { controlled clinical } \\
\text { trials }\end{array}$ & 2016 & IJMOS & $\begin{array}{l}\text { University of } \\
\text { L'Aquila }\end{array}$ & 33 \\
\hline 76 & $\begin{array}{l}\text { Al-Moraissi } \\
\text { EA et al. }\end{array}$ & $\begin{array}{l}\text { A systematic review } \\
\text { and meta-analysis } \\
\text { of the clinical } \\
\text { outcomes for } \\
\text { various surgical } \\
\text { modalities in the } \\
\text { management of } \\
\text { temporomandibular } \\
\text { joint ankylosis }\end{array}$ & 2015 & IJMOS & $\begin{array}{l}\text { Thamar } \\
\text { University }\end{array}$ & 33 \\
\hline 77 & $\begin{array}{l}\text { Haas OL et } \\
\text { al. }\end{array}$ & $\begin{array}{l}\text { Computer-aided } \\
\text { planning in } \\
\text { orthognathic } \\
\text { surgery-systematic } \\
\text { review }\end{array}$ & 2015 & IJMOS & $\begin{array}{l}\text { Pontificial } \\
\text { Catholic } \\
\text { University of Rio } \\
\text { Grande do Sul }\end{array}$ & 33 \\
\hline 78 & $\begin{array}{l}\text { Lovelace TL } \\
\text { et al. }\end{array}$ & $\begin{array}{l}\text { Management of } \\
\text { radiotherapy- } \\
\text { induced salivary } \\
\text { hypofunction and } \\
\text { consequent } \\
\text { xerostomia in } \\
\text { patients with oral or } \\
\text { head and neck } \\
\text { cancer: meta- } \\
\text { analysis and } \\
\text { literature review }\end{array}$ & 2014 & TRIPLEO & $\begin{array}{l}\text { Medical } \\
\text { University of } \\
\text { South Carolina }\end{array}$ & 33 \\
\hline 79 & $\begin{array}{l}\text { Saltaji H et } \\
\text { al. }\end{array}$ & $\begin{array}{l}\text { Maxillary } \\
\text { Advancement With } \\
\text { Conventional } \\
\text { Orthognathic } \\
\text { Surgery in Patients } \\
\text { With Cleft Lip and } \\
\text { Palate: Is It a Stable } \\
\text { Technique? }\end{array}$ & 2012 & JOMS & $\begin{array}{l}\text { University of } \\
\text { Alberta }\end{array}$ & 32 \\
\hline 80 & $\begin{array}{l}\text { Tarsitano A } \\
\text { et al. }\end{array}$ & $\begin{array}{l}\text { Mandibular } \\
\text { reconstructions } \\
\text { using computer- } \\
\text { aided } \\
\text { design/computer- } \\
\text { aided } \\
\text { manufacturing: A } \\
\text { systematic review } \\
\text { of a defect-based } \\
\text { reconstructive } \\
\text { algorithm }\end{array}$ & 2015 & JCMS & $\begin{array}{l}\text { University of } \\
\text { Bologna }\end{array}$ & 32 \\
\hline
\end{tabular}




\begin{tabular}{|c|c|c|c|c|c|c|}
\hline 81 & $\begin{array}{l}\text { Adeyemo } \\
\text { WL, Akadiri } \\
\text { OA }\end{array}$ & $\begin{array}{l}\text { A systematic review } \\
\text { of the diagnostic } \\
\text { role of } \\
\text { ultrasonography in } \\
\text { maxillofacial } \\
\text { fractures }\end{array}$ & 2011 & IJMOS & $\begin{array}{l}\text { University of } \\
\text { Lagos }\end{array}$ & 32 \\
\hline 82 & $\begin{array}{l}\text { Agbaje JO } \\
\text { et al. }\end{array}$ & $\begin{array}{l}\text { Systematic review } \\
\text { of the incidence of } \\
\text { inferior alveolar } \\
\text { nerve injury in } \\
\text { bilateral sagittal } \\
\text { split osteotomy and } \\
\text { the assessment of } \\
\text { neurosensory } \\
\text { disturbances }\end{array}$ & 2015 & IJMOS & $\begin{array}{l}\text { Catholic } \\
\text { University Leuven }\end{array}$ & 31 \\
\hline 83 & $\begin{array}{l}\text { Hsieh YJ, } \\
\text { Liao YF }\end{array}$ & $\begin{array}{l}\text { Effects of } \\
\text { maxillomandibular } \\
\text { advancement on the } \\
\text { upper airway and } \\
\text { surrounding } \\
\text { structures in } \\
\text { patients with } \\
\text { obstructive sleep } \\
\text { apnoea: a } \\
\text { systematic review }\end{array}$ & 2013 & BJOMS & $\begin{array}{l}\text { Chang Gung } \\
\text { Memorial } \\
\text { Hospital }\end{array}$ & 31 \\
\hline 84 & $\begin{array}{l}\text { Molina- } \\
\text { Solana R et } \\
\text { al. }\end{array}$ & $\begin{array}{l}\text { Current concepts on } \\
\text { the effect of } \\
\text { environmental } \\
\text { factors on cleft lip } \\
\text { and palate }\end{array}$ & 2013 & IJMOS & $\begin{array}{l}\text { University of } \\
\text { Seville }\end{array}$ & 31 \\
\hline 85 & $\begin{array}{l}\text { Al-Moraissi } \\
\text { EA, Ellis E }\end{array}$ & $\begin{array}{l}\text { What Method for } \\
\text { Management of } \\
\text { Unilateral } \\
\text { Mandibular Angle } \\
\text { Fractures Has the } \\
\text { Lowest Rate of } \\
\text { Postoperative } \\
\text { Complications? A } \\
\text { Systematic Review } \\
\text { and Meta-Analysis }\end{array}$ & 2014 & JOMS & $\begin{array}{l}\text { Thamar } \\
\text { University }\end{array}$ & 30 \\
\hline 86 & $\begin{array}{l}\text { Al-Moraissi } \\
\text { EA et al. }\end{array}$ & $\begin{array}{l}\text { What surgical } \\
\text { treatment has the } \\
\text { lowest recurrence } \\
\text { rate following the } \\
\text { management of } \\
\text { keratocystic } \\
\text { odontogenic } \\
\text { tumor?: A large } \\
\text { systematic review } \\
\text { and meta-analysis }\end{array}$ & 2017 & JCMS & $\begin{array}{l}\text { Thamar } \\
\text { University }\end{array}$ & 30 \\
\hline 87 & $\begin{array}{l}\text { Antonoglou } \\
\text { GN, Sandor } \\
\text { GK }\end{array}$ & $\begin{array}{l}\text { Recurrence rates of } \\
\text { intraosseous } \\
\text { ameloblastomas of } \\
\text { the jaws: A } \\
\text { systematic review } \\
\text { of conservative }\end{array}$ & 2015 & JCMS & University of Oulu & 30 \\
\hline
\end{tabular}




\begin{tabular}{|c|c|c|c|c|c|c|}
\hline & & $\begin{array}{l}\text { versus aggressive } \\
\text { treatment } \\
\text { approaches and } \\
\text { meta-analysis of } \\
\text { non-randomized } \\
\text { studies }\end{array}$ & & & & \\
\hline 88 & $\begin{array}{l}\text { Shah KSV, } \\
\text { Ethunandan } \\
\text { M }\end{array}$ & $\begin{array}{l}\text { Tumour seeding } \\
\text { after fine-needle } \\
\text { aspiration and core } \\
\text { biopsy of the head } \\
\text { and neck - a } \\
\text { systematic review }\end{array}$ & 2016 & BJOMS & $\begin{array}{l}\text { University } \\
\text { Hospitals } \\
\text { Southampton }\end{array}$ & 29 \\
\hline 89 & Breik $O$ et al. & $\begin{array}{l}\text { Mandibular } \\
\text { distraction } \\
\text { osteogenesis for the } \\
\text { management of } \\
\text { upper airway } \\
\text { obstruction in } \\
\text { children with } \\
\text { micrognathia: a } \\
\text { systematic review }\end{array}$ & 2016 & IJMOS & $\begin{array}{l}\text { Royal Melbourne } \\
\text { Hospital }\end{array}$ & 29 \\
\hline 90 & $\begin{array}{l}\text { Clementini } \\
M \text { et al. }\end{array}$ & $\begin{array}{l}\text { Immediate versus } \\
\text { delayed positioning } \\
\text { of dental implants } \\
\text { in guided bone } \\
\text { regeneration or } \\
\text { onlay graft } \\
\text { regenerated areas: a } \\
\text { systematic review }\end{array}$ & 2013 & IJMOS & $\begin{array}{l}\text { University "Tor } \\
\text { Vergata" }\end{array}$ & 29 \\
\hline 91 & $\begin{array}{l}\text { Saltaji H et } \\
\text { al. }\end{array}$ & $\begin{array}{l}\text { Le Fort III } \\
\text { Distraction } \\
\text { Osteogenesis } \\
\text { Versus } \\
\text { Conventional Le } \\
\text { Fort III Osteotomy in } \\
\text { Correction of } \\
\text { Syndromic } \\
\text { Midfacial } \\
\text { Hypoplasia: A } \\
\text { Systematic Review }\end{array}$ & 2014 & JOMS & $\begin{array}{l}\text { University of } \\
\text { Alberta }\end{array}$ & 28 \\
\hline 92 & $\begin{array}{l}\text { Moraschini } \\
\text { V et al. }\end{array}$ & $\begin{array}{l}\text { Effect of } \\
\text { autologous platelet } \\
\text { concentrates for } \\
\text { alveolar socket } \\
\text { preservation: a } \\
\text { systematic review }\end{array}$ & 2015 & IJMOS & $\begin{array}{l}\text { Fluminense } \\
\text { Federal University }\end{array}$ & 28 \\
\hline 93 & $\begin{array}{l}\text { Almeida } \\
\text { RDC; et al. }\end{array}$ & $\begin{array}{l}\text { Recurrence rate } \\
\text { following treatment } \\
\text { for primary } \\
\text { multicystic } \\
\text { ameloblastoma: } \\
\text { systematic review } \\
\text { and meta-analysis }\end{array}$ & 2016 & IJMOS & $\begin{array}{l}\text { University of } \\
\text { Pernambuco }\end{array}$ & 27 \\
\hline 94 & $\begin{array}{l}\text { Langton S } \\
\text { et al. }\end{array}$ & $\begin{array}{l}\text { Two-week rule in } \\
\text { head and neck }\end{array}$ & 2016 & BJOMS & $\begin{array}{l}\text { Royal Blackburn } \\
\text { Hospital }\end{array}$ & 27 \\
\hline
\end{tabular}


cancer 2000-14: a

systematic review

\begin{tabular}{|c|c|c|c|c|c|c|}
\hline 95 & $\begin{array}{l}\text { Atienza G, } \\
\text { Lopez- } \\
\text { Cedrun JL }\end{array}$ & $\begin{array}{l}\text { Management of } \\
\text { obstructive salivary } \\
\text { disorders by } \\
\text { sialendoscopy: a } \\
\text { systematic review }\end{array}$ & 2015 & BJOMS & $\begin{array}{l}\text { Galician Agency } \\
\text { for Health } \\
\text { Technology } \\
\text { Assessment }\end{array}$ & 27 \\
\hline 96 & $\begin{array}{l}\text { Taylor J et } \\
\text { al. }\end{array}$ & $\begin{array}{l}\text { World Workshop on } \\
\text { Oral Medicine VI: a } \\
\text { systematic review } \\
\text { of the treatment of } \\
\text { mucous membrane } \\
\text { pemphigoid }\end{array}$ & 2015 & TRIPLEO & $\begin{array}{l}\text { New York } \\
\text { University College } \\
\text { of Dentistry }\end{array}$ & 27 \\
\hline 97 & $\begin{array}{l}\text { Voulgarakis } \\
\text { A et al. }\end{array}$ & $\begin{array}{l}\text { Outcomes of } \\
\text { implants placed } \\
\text { with three different } \\
\text { flapless surgical } \\
\text { procedures: A } \\
\text { systematic review }\end{array}$ & 2014 & IJMOS & $\begin{array}{l}\text { University } \\
\text { Hospital of } \\
\text { Freiburg }\end{array}$ & 27 \\
\hline 98 & Li CJ et al. & $\begin{array}{l}\text { Ultrasonography for } \\
\text { Detection of Disc } \\
\text { Displacement of } \\
\text { Temporomandibular } \\
\text { Joint: A Systematic } \\
\text { Review and Meta- } \\
\text { Analysis }\end{array}$ & 2012 & JOMS & $\begin{array}{l}\text { Sichuan } \\
\text { University }\end{array}$ & 27 \\
\hline 99 & $\begin{array}{l}\text { Leung YY et } \\
\text { al. }\end{array}$ & $\begin{array}{l}\text { Treatment } \\
\text { Modalities of } \\
\text { Neurosensory } \\
\text { Deficit After Lower } \\
\text { Third Molar Surgery: } \\
\text { A Systematic } \\
\text { Review }\end{array}$ & 2012 & JOMS & $\begin{array}{l}\text { Prince Philip } \\
\text { Dental Hospital }\end{array}$ & 27 \\
\hline 100 & $\begin{array}{l}\text { Joss CU et } \\
\text { al. }\end{array}$ & $\begin{array}{l}\text { Soft Tissue Profile } \\
\text { Changes After } \\
\text { Bilateral Sagittal } \\
\text { Split Osteotomy for } \\
\text { Mandibular } \\
\text { Setback: A } \\
\text { Systematic Review }\end{array}$ & 2010 & JOMS & $\begin{array}{l}\text { Radboud } \\
\text { University }\end{array}$ & 27 \\
\hline
\end{tabular}

\section{Figures}




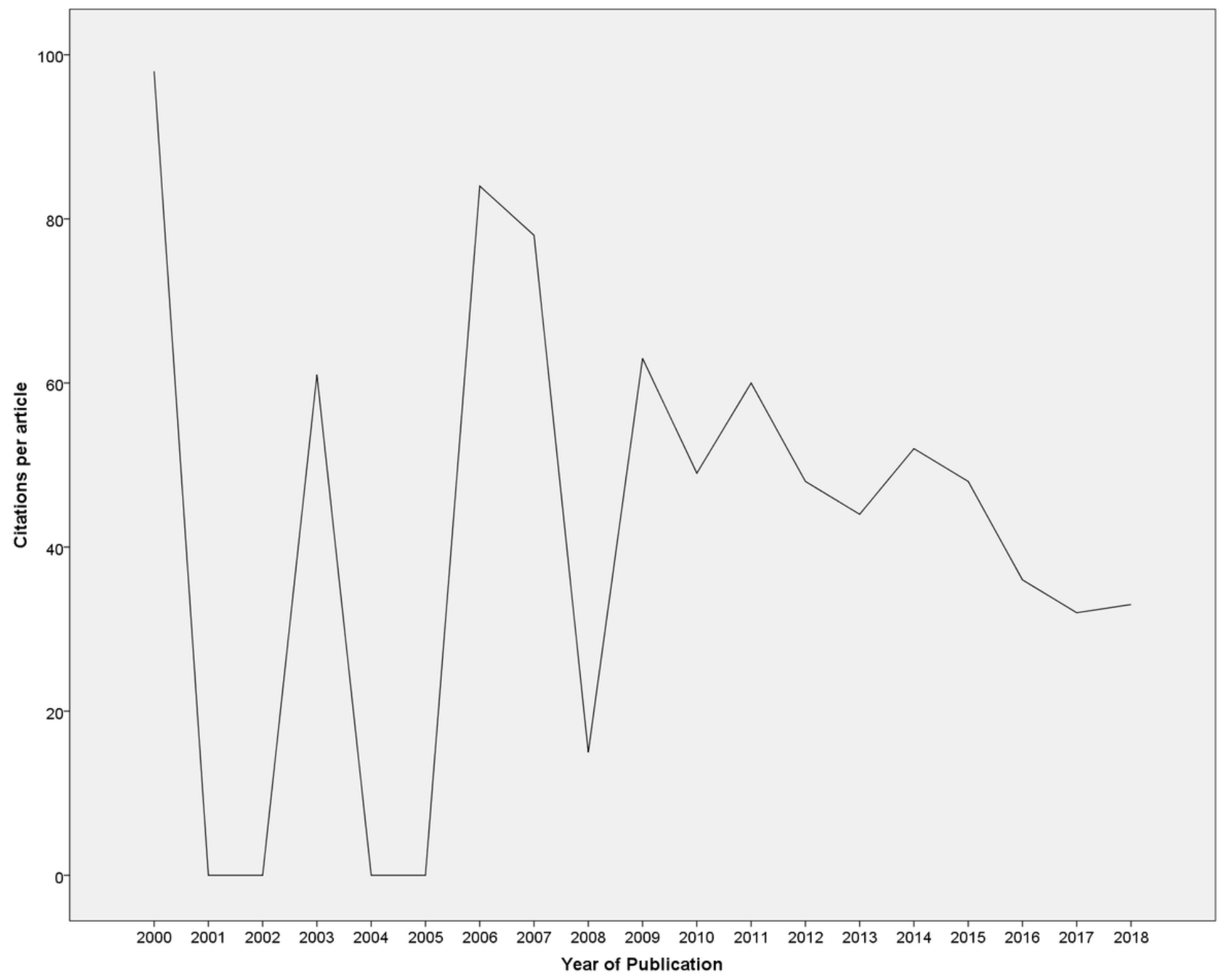

Figure 1

Number of citations per systematic review by year in OMFS 


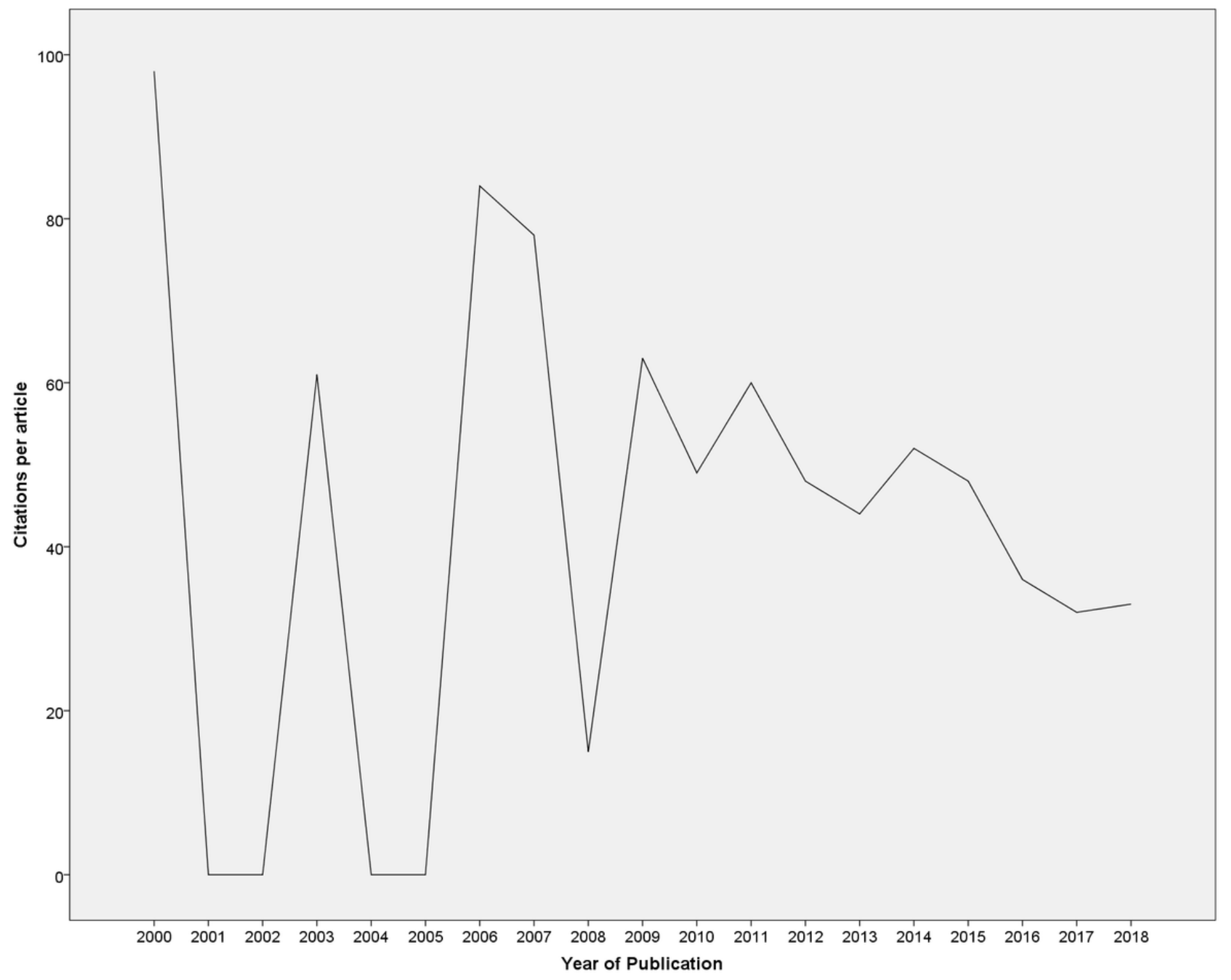

Figure 1

Number of citations per systematic review by year in OMFS 


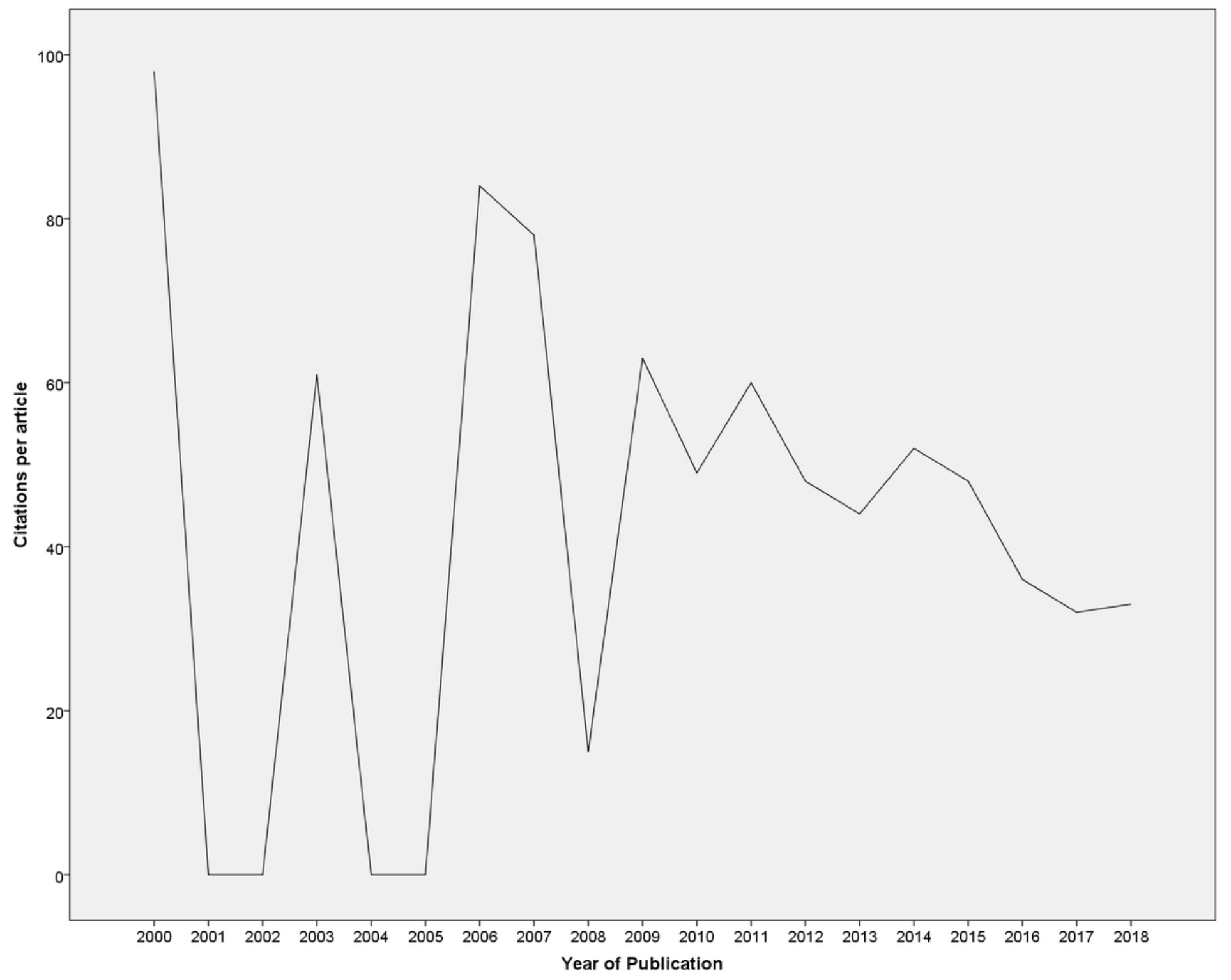

Figure 1

Number of citations per systematic review by year in OMFS 


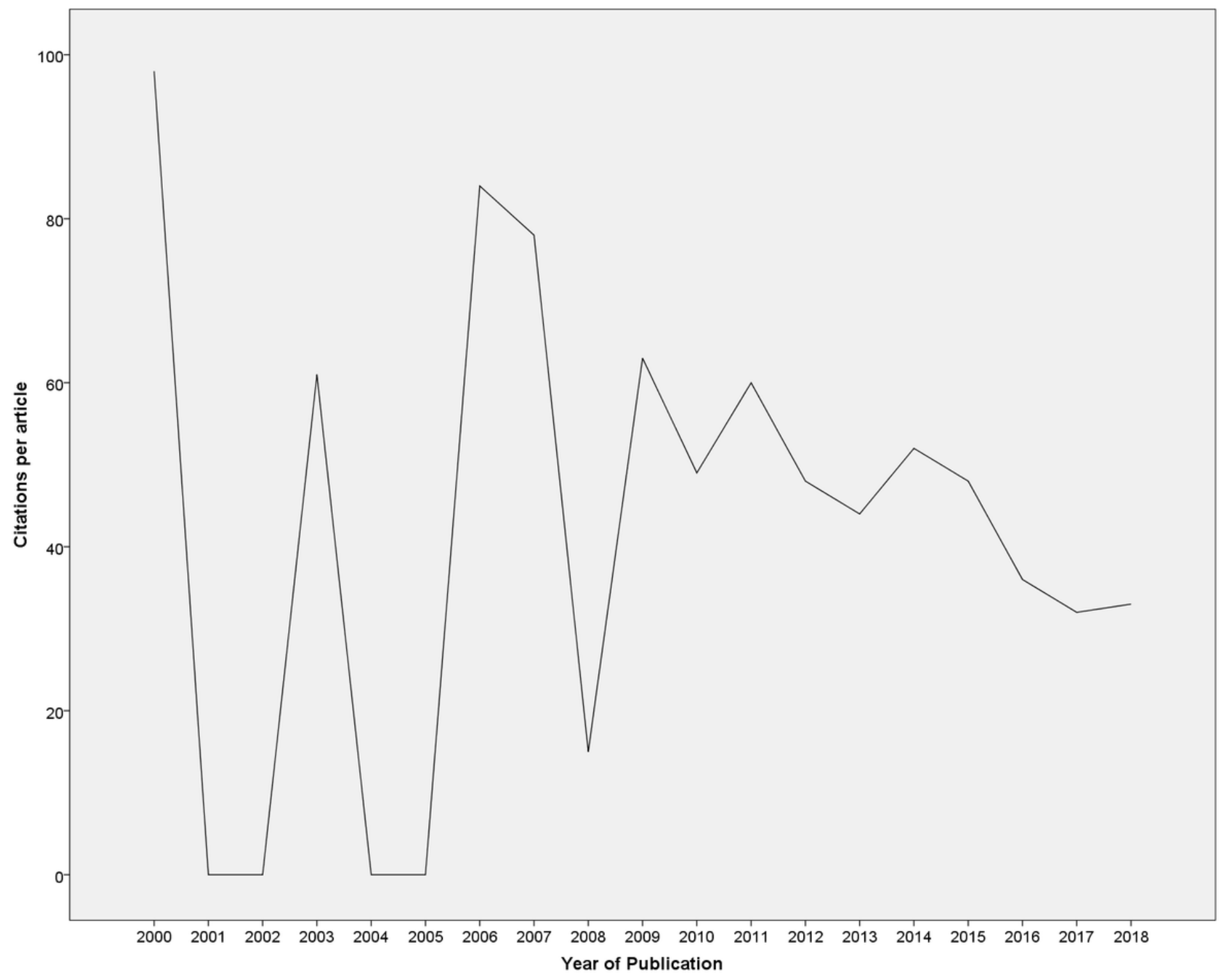

Figure 1

Number of citations per systematic review by year in OMFS 


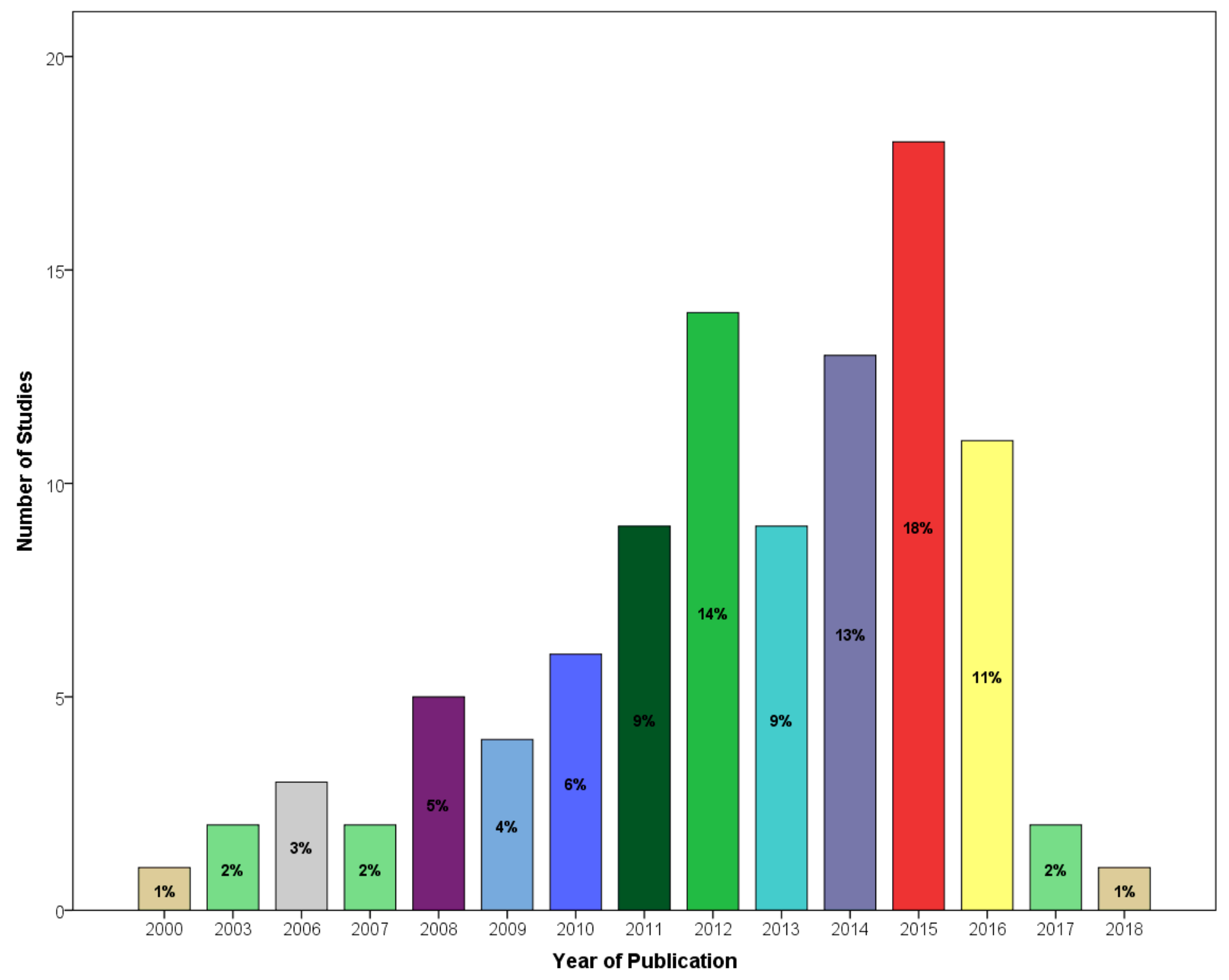

Figure 2

Time-pattern distribution of the 100 most cited systematic reviews on oral and maxillofacial surgery 


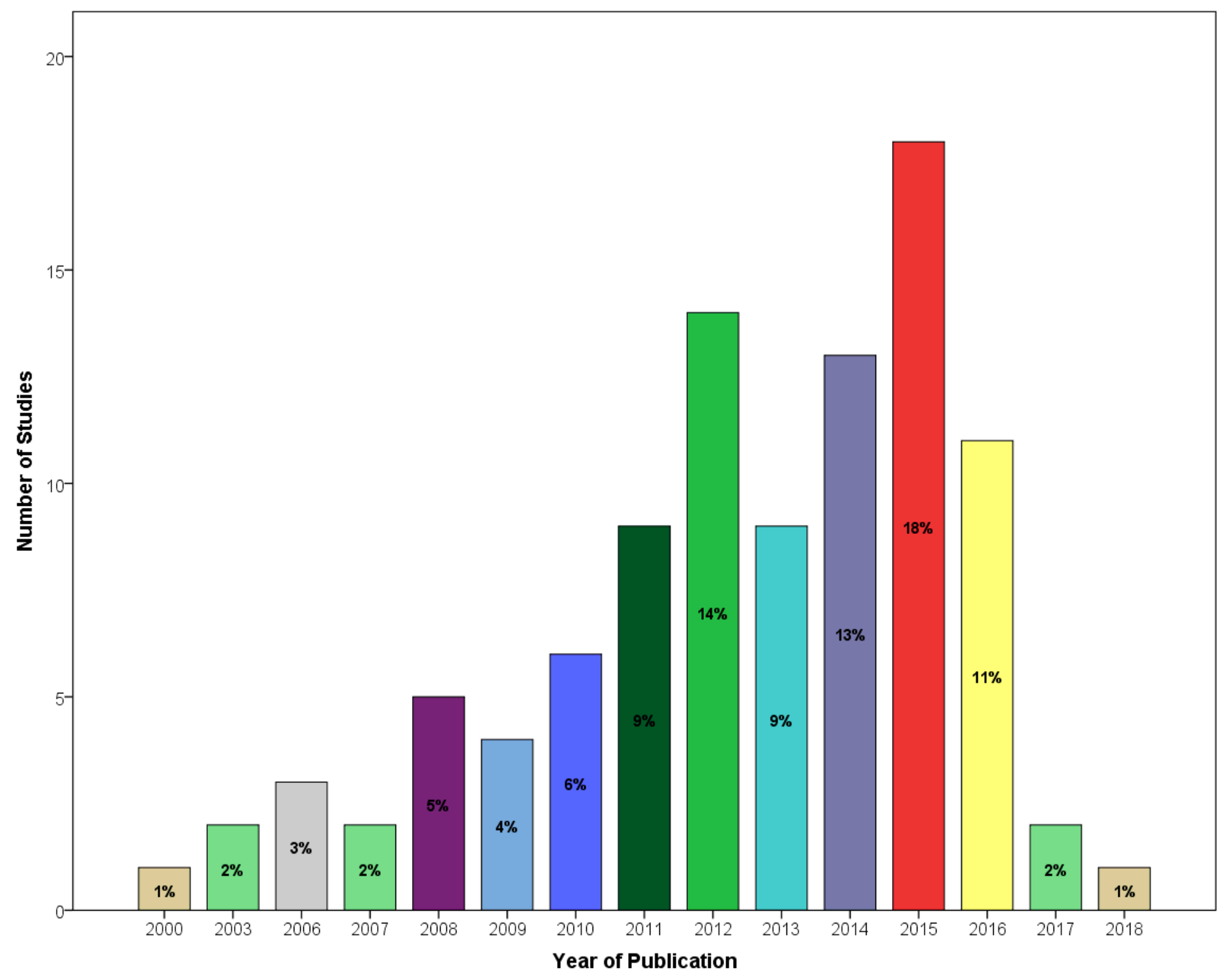

Figure 2

Time-pattern distribution of the 100 most cited systematic reviews on oral and maxillofacial surgery 


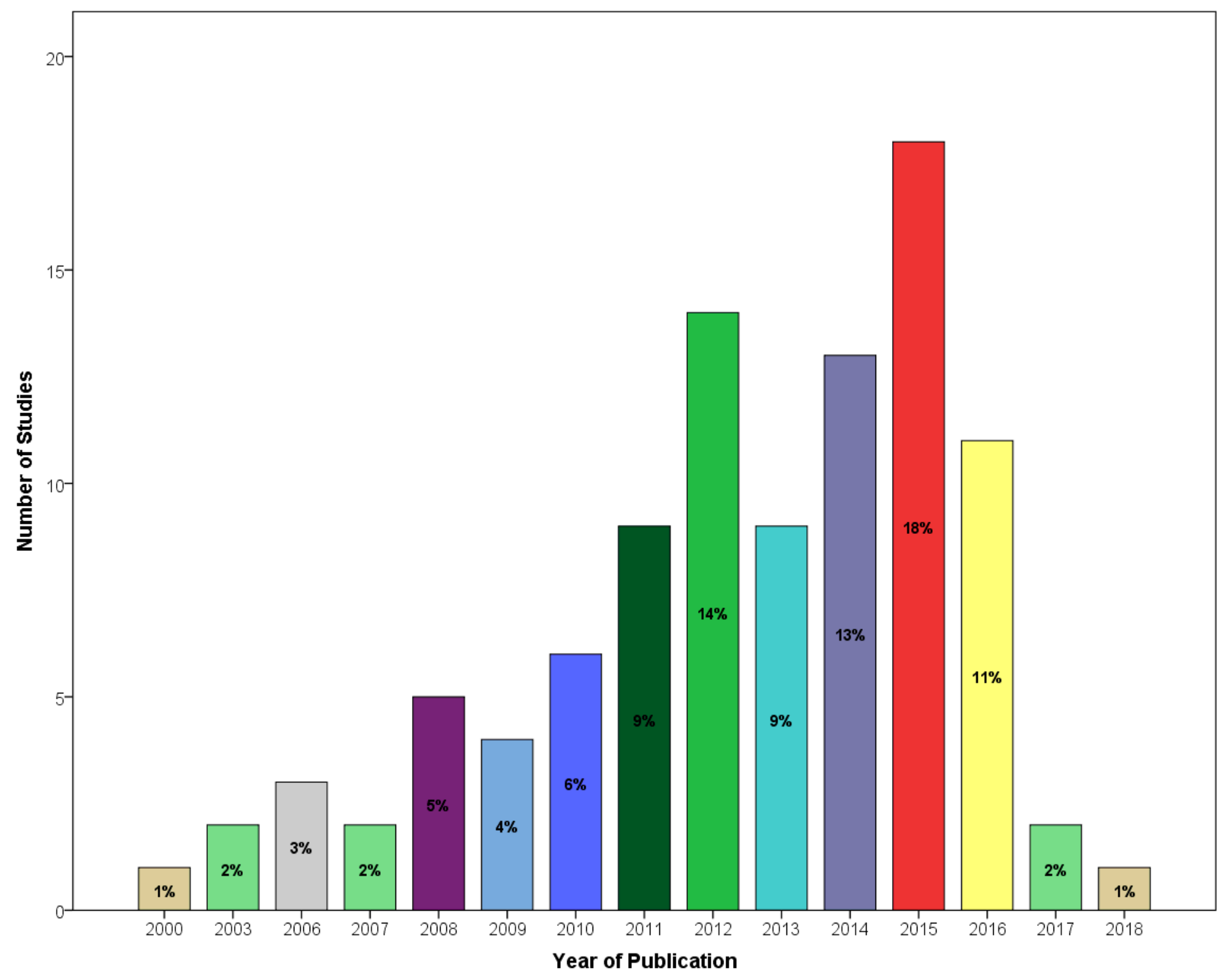

Figure 2

Time-pattern distribution of the 100 most cited systematic reviews on oral and maxillofacial surgery 


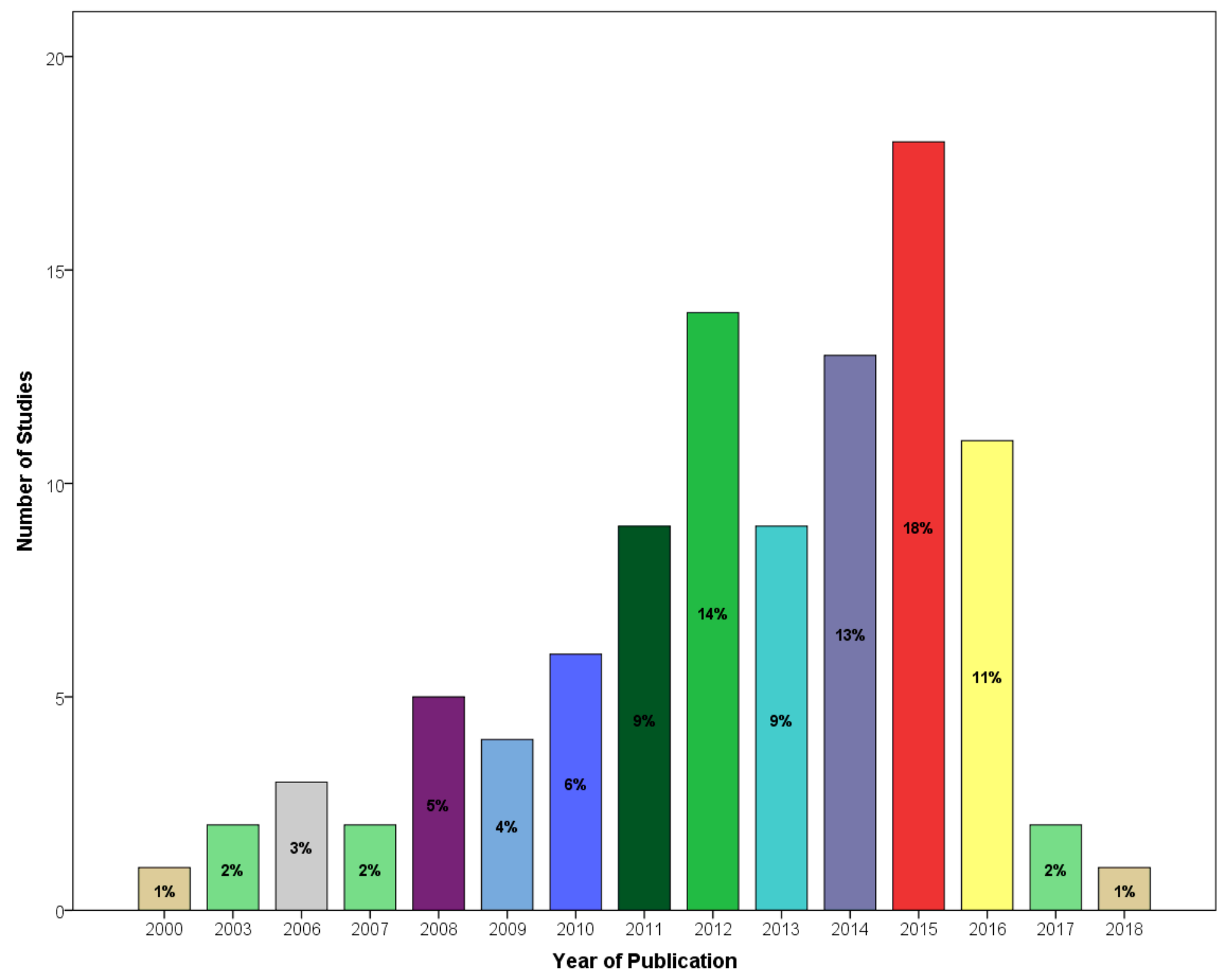

Figure 2

Time-pattern distribution of the 100 most cited systematic reviews on oral and maxillofacial surgery 


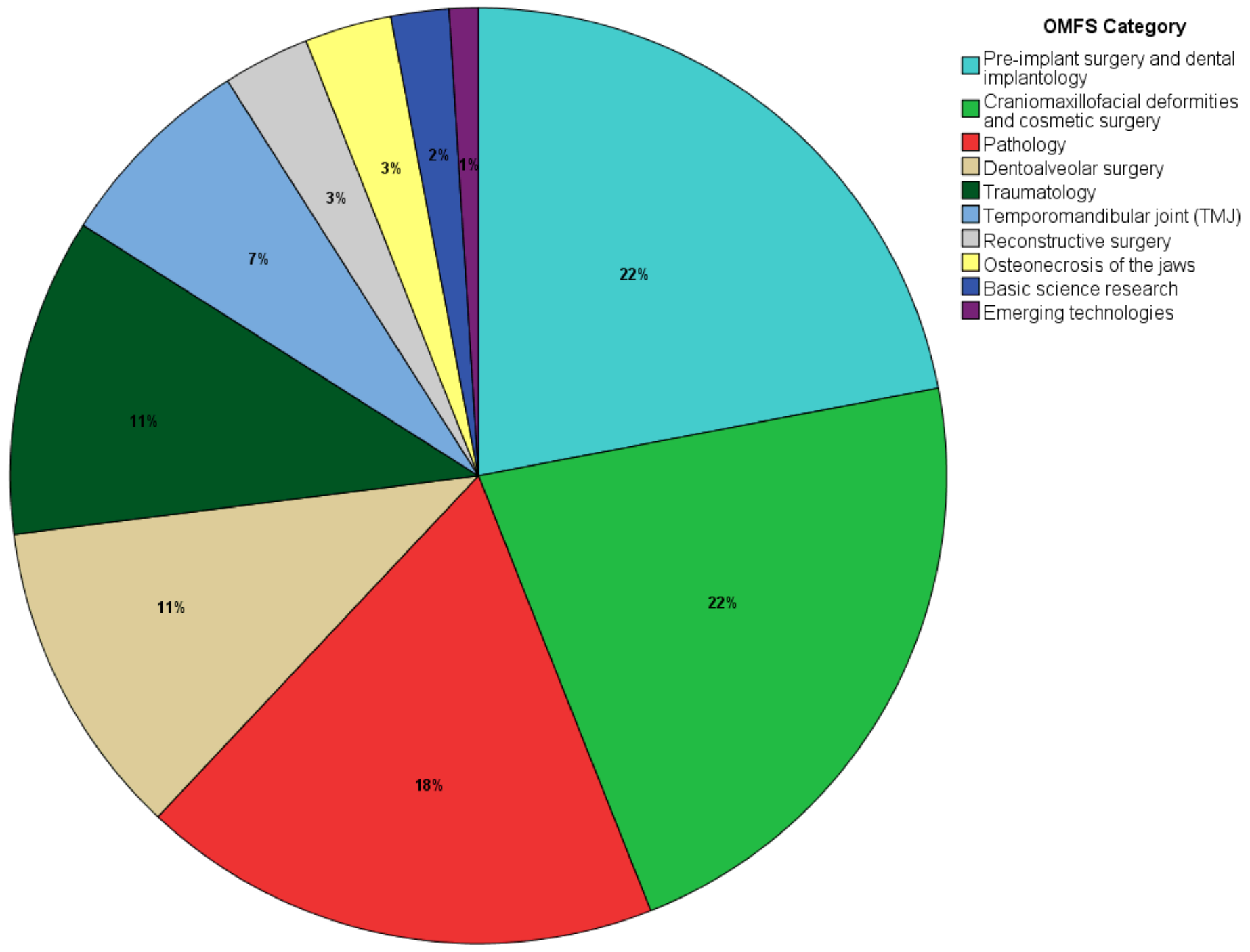

Figure 3

Topics covered among the 100 most cited systematic reviews on oral and maxillofacial surgery 


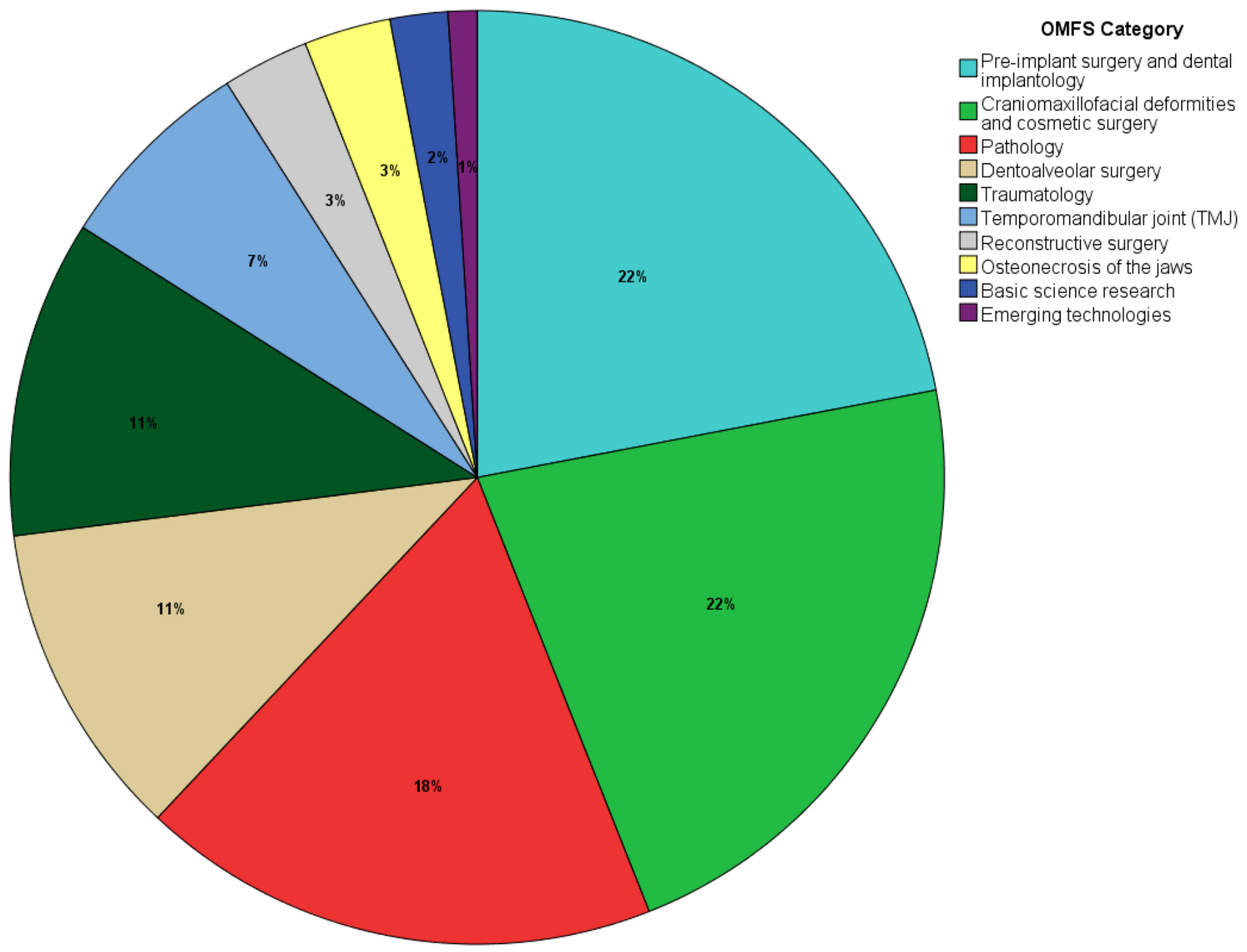

Figure 3

Topics covered among the 100 most cited systematic reviews on oral and maxillofacial surgery 


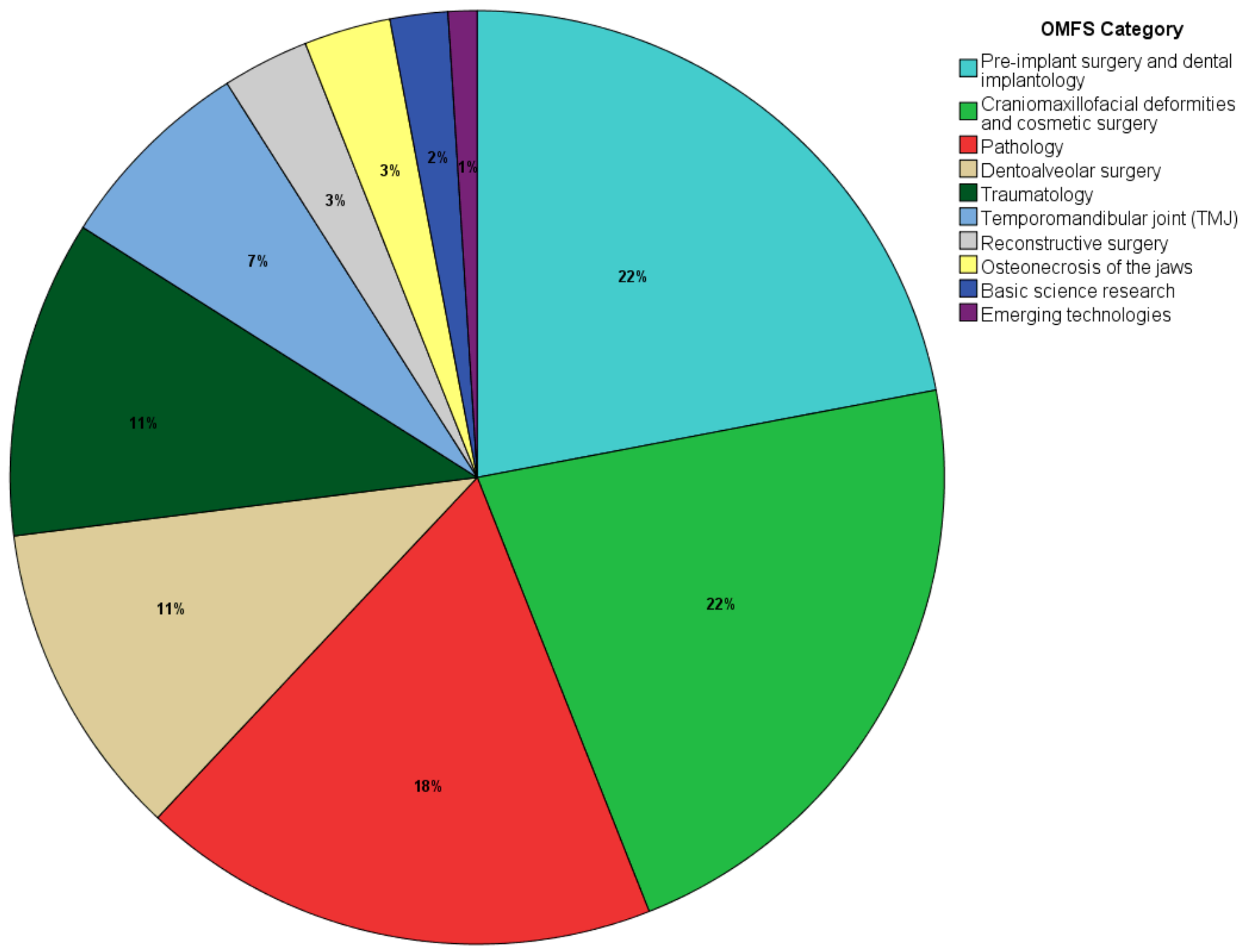

Figure 3

Topics covered among the 100 most cited systematic reviews on oral and maxillofacial surgery 


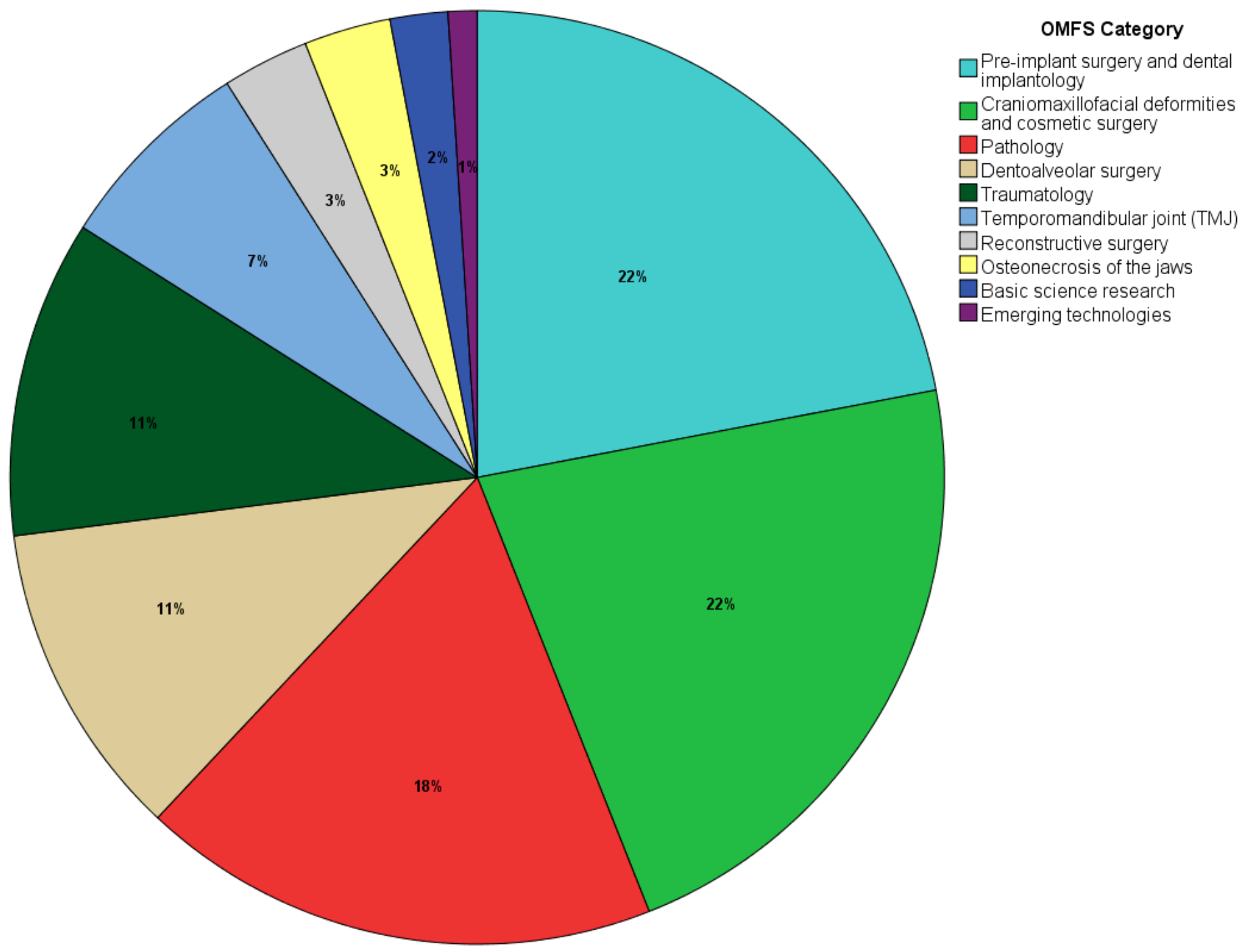

Figure 3

Topics covered among the 100 most cited systematic reviews on oral and maxillofacial surgery 


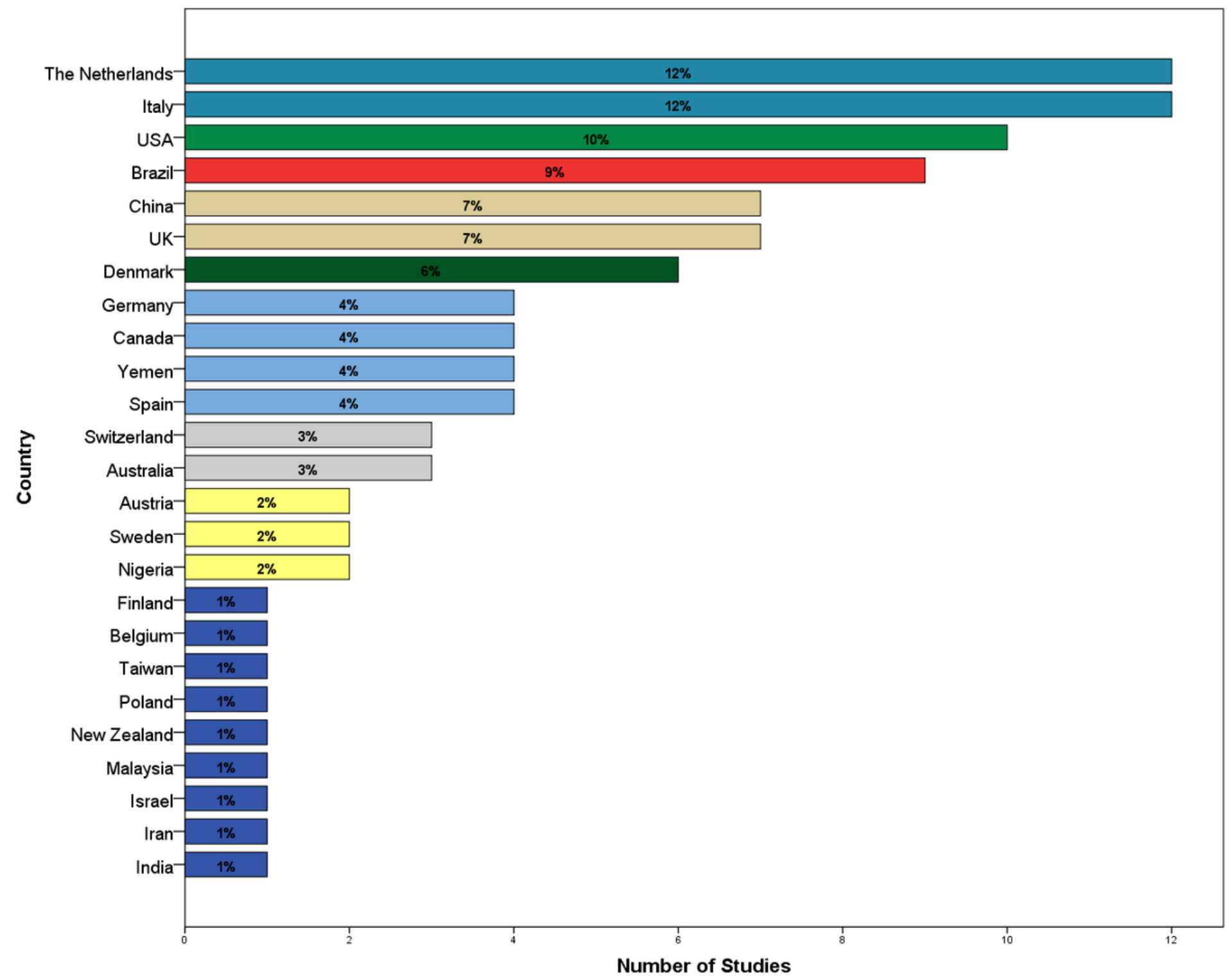

\section{Figure 4}

Country of author's institute for 100 most cited systematic reviews 


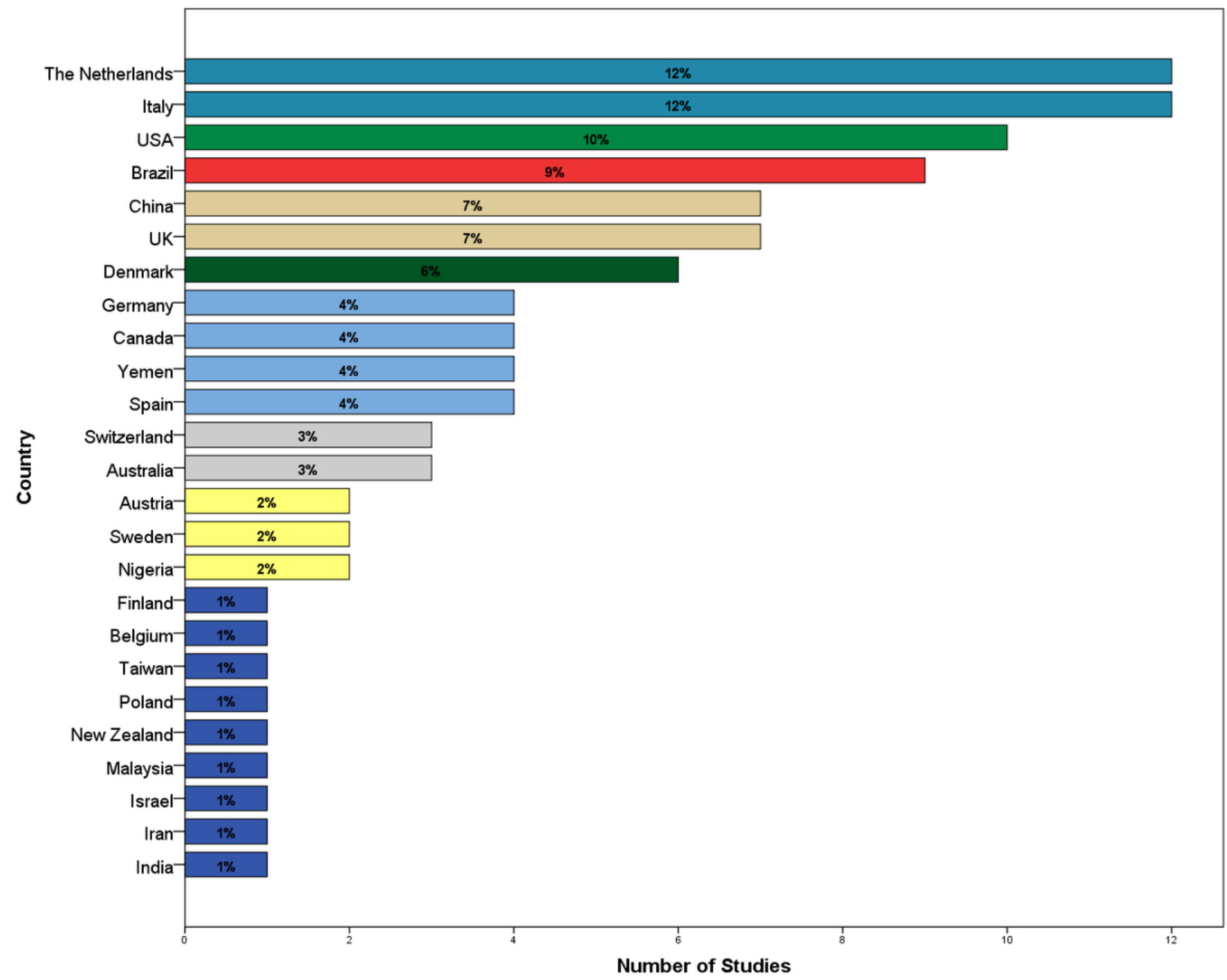

\section{Figure 4}

Country of author's institute for 100 most cited systematic reviews 


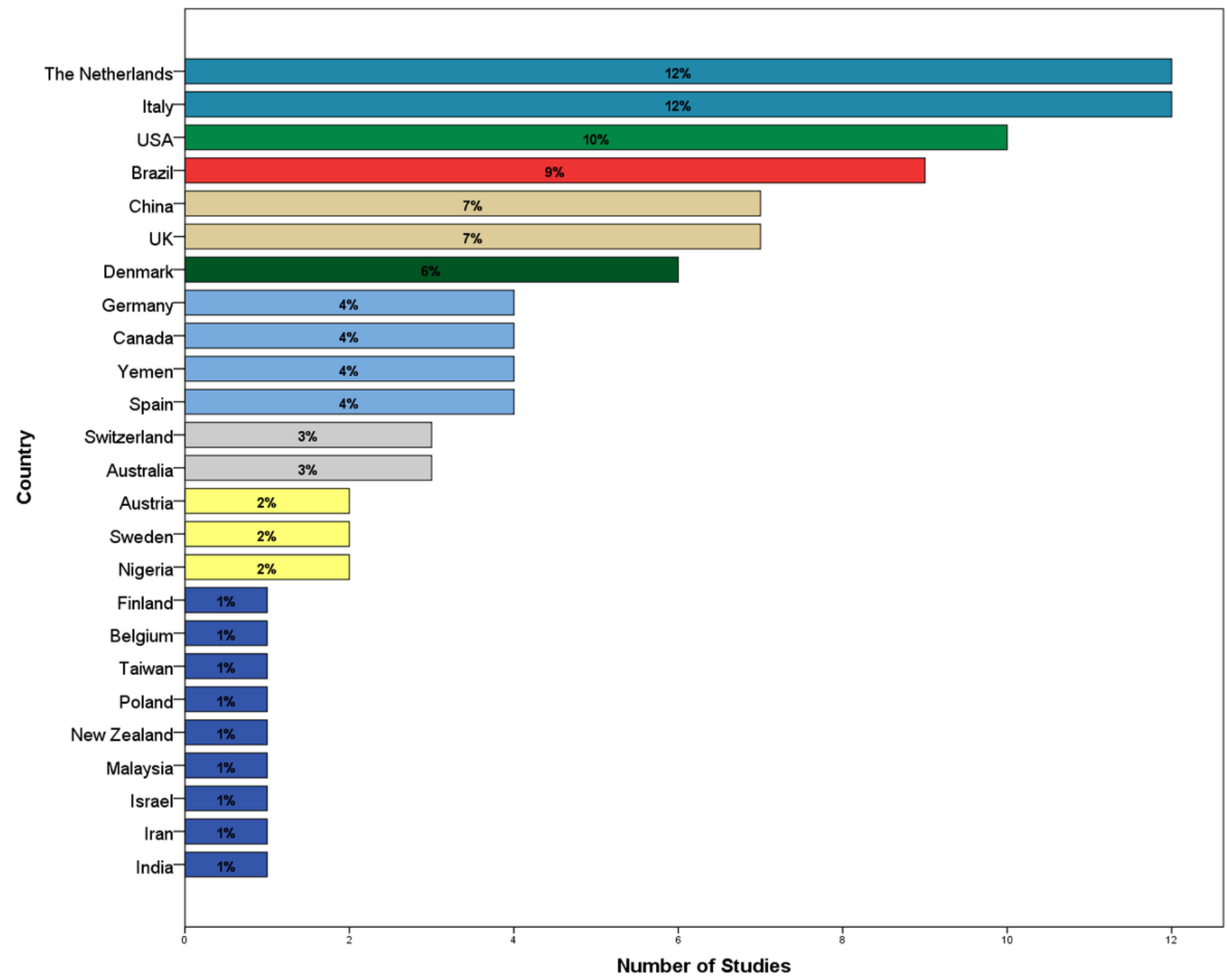

\section{Figure 4}

Country of author's institute for 100 most cited systematic reviews 


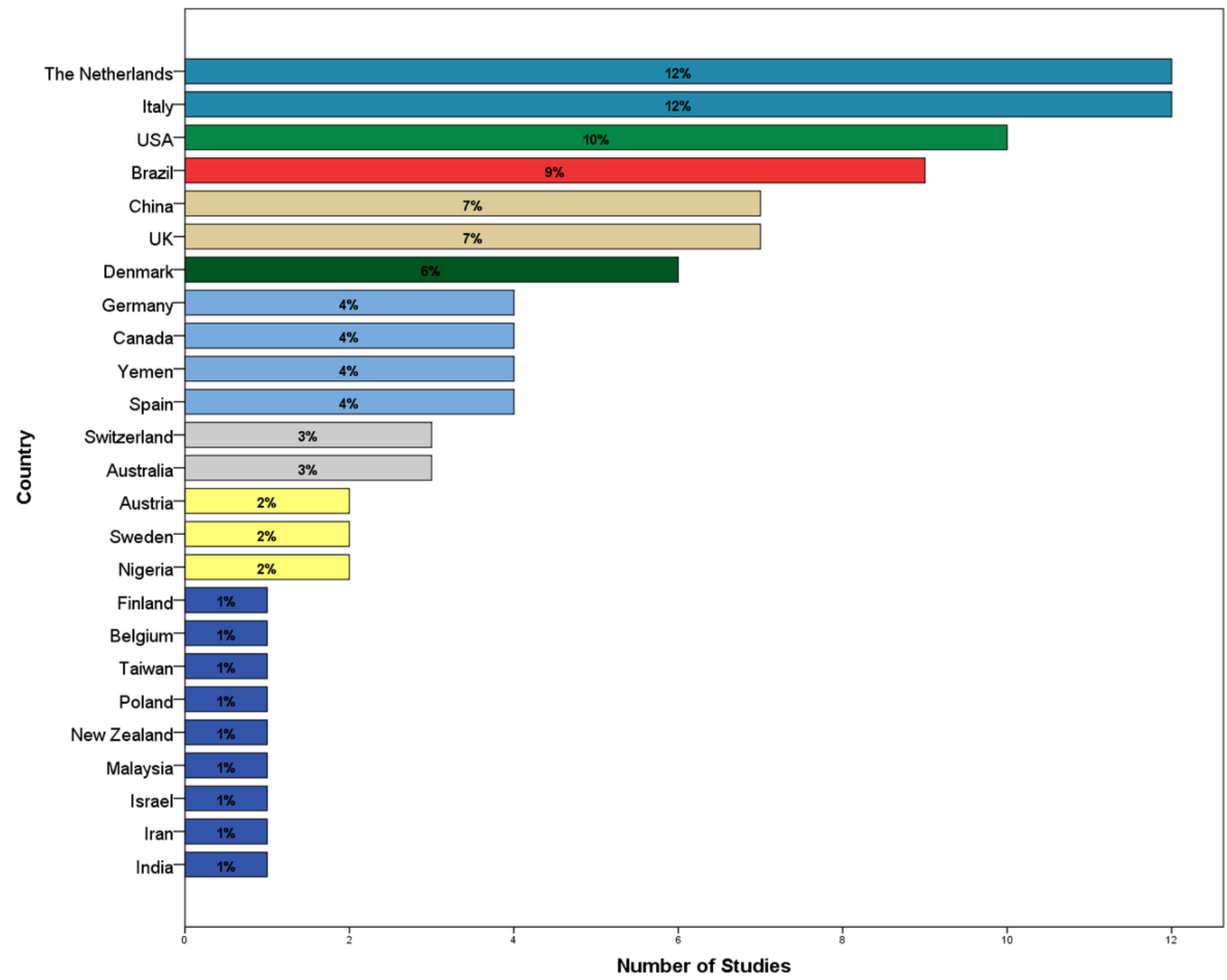

\section{Figure 4}

Country of author's institute for 100 most cited systematic reviews 


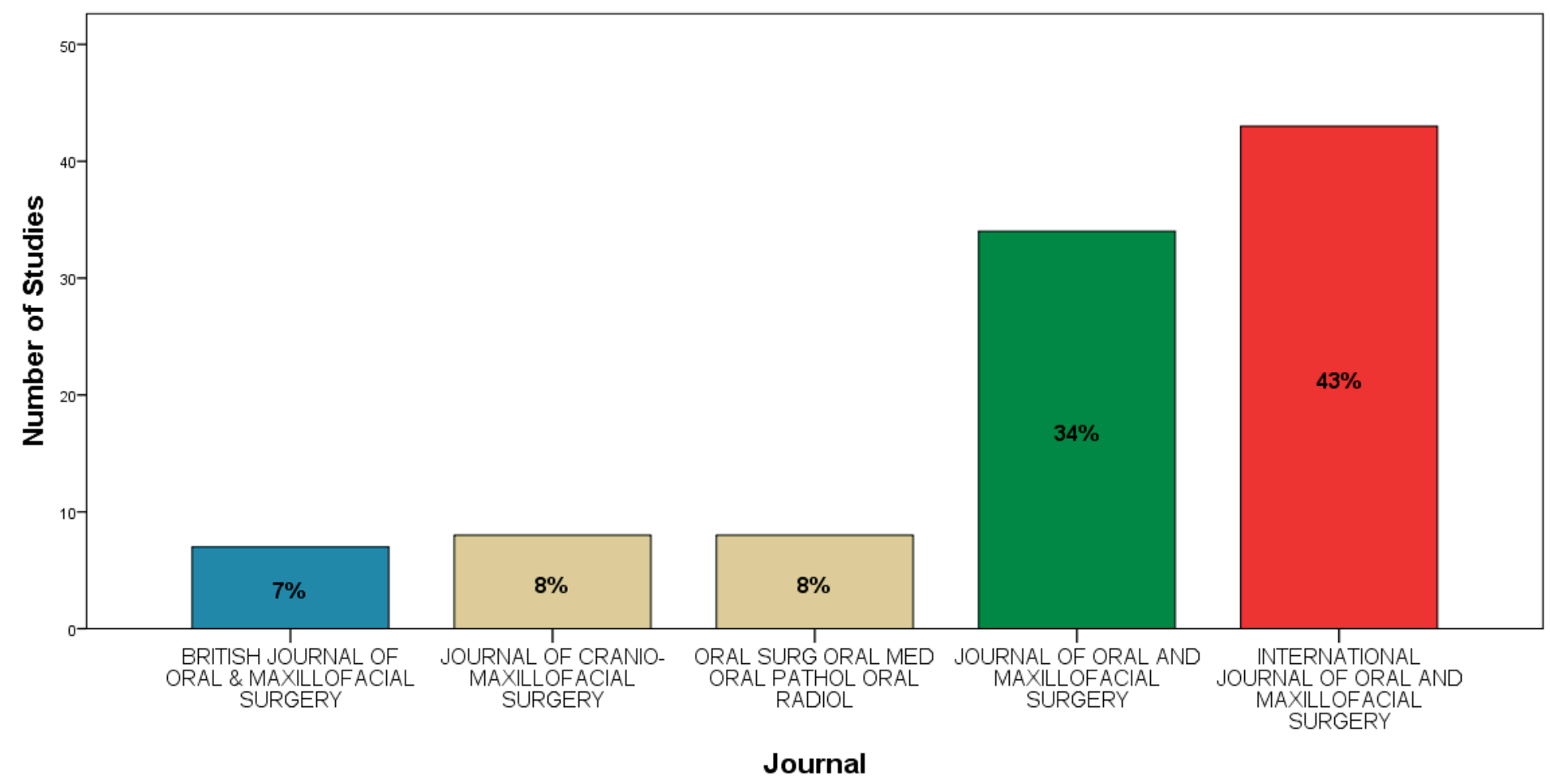

Figure 5

Most frequently cited journal

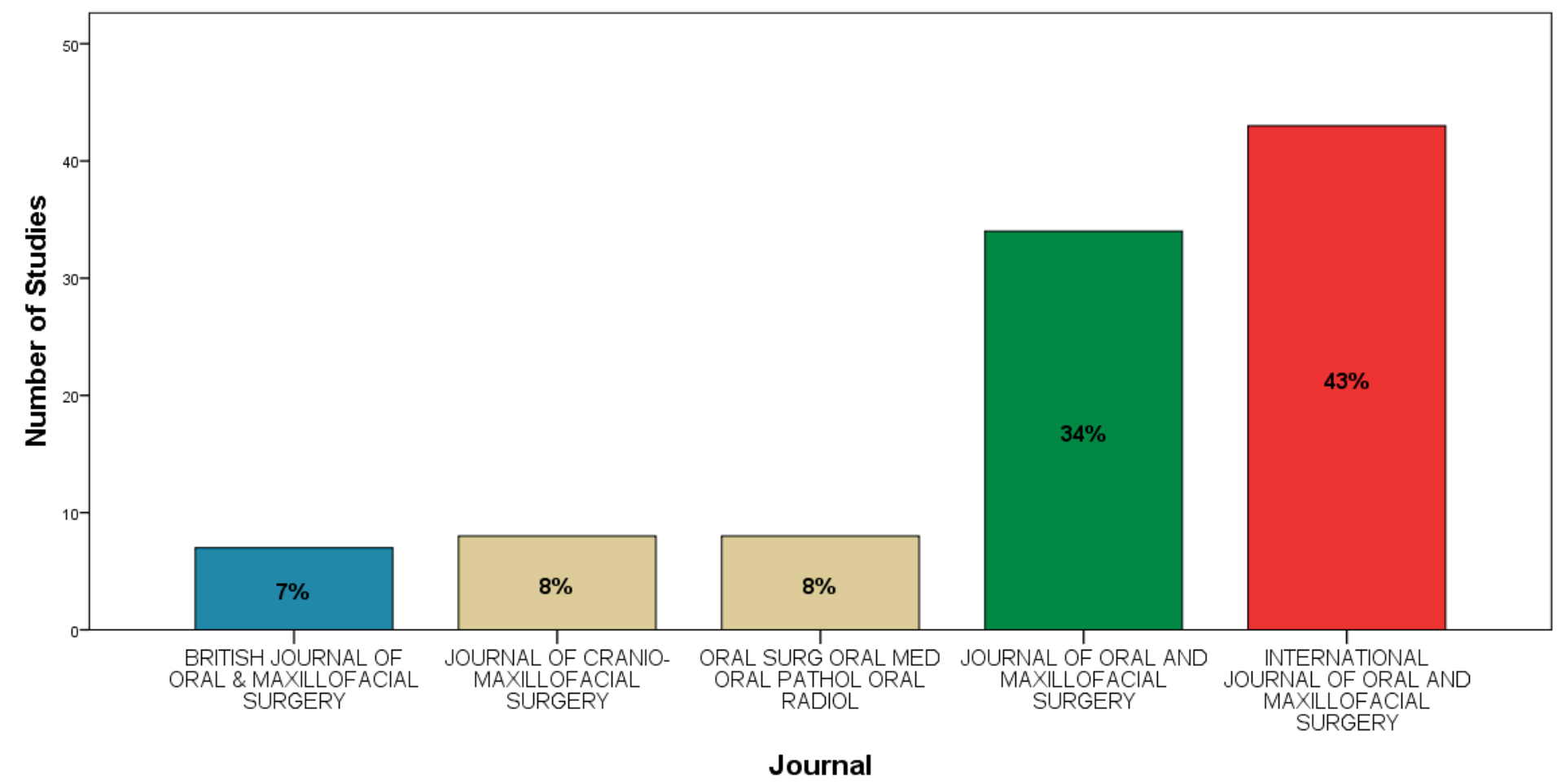

Figure 5

Most frequently cited journal 


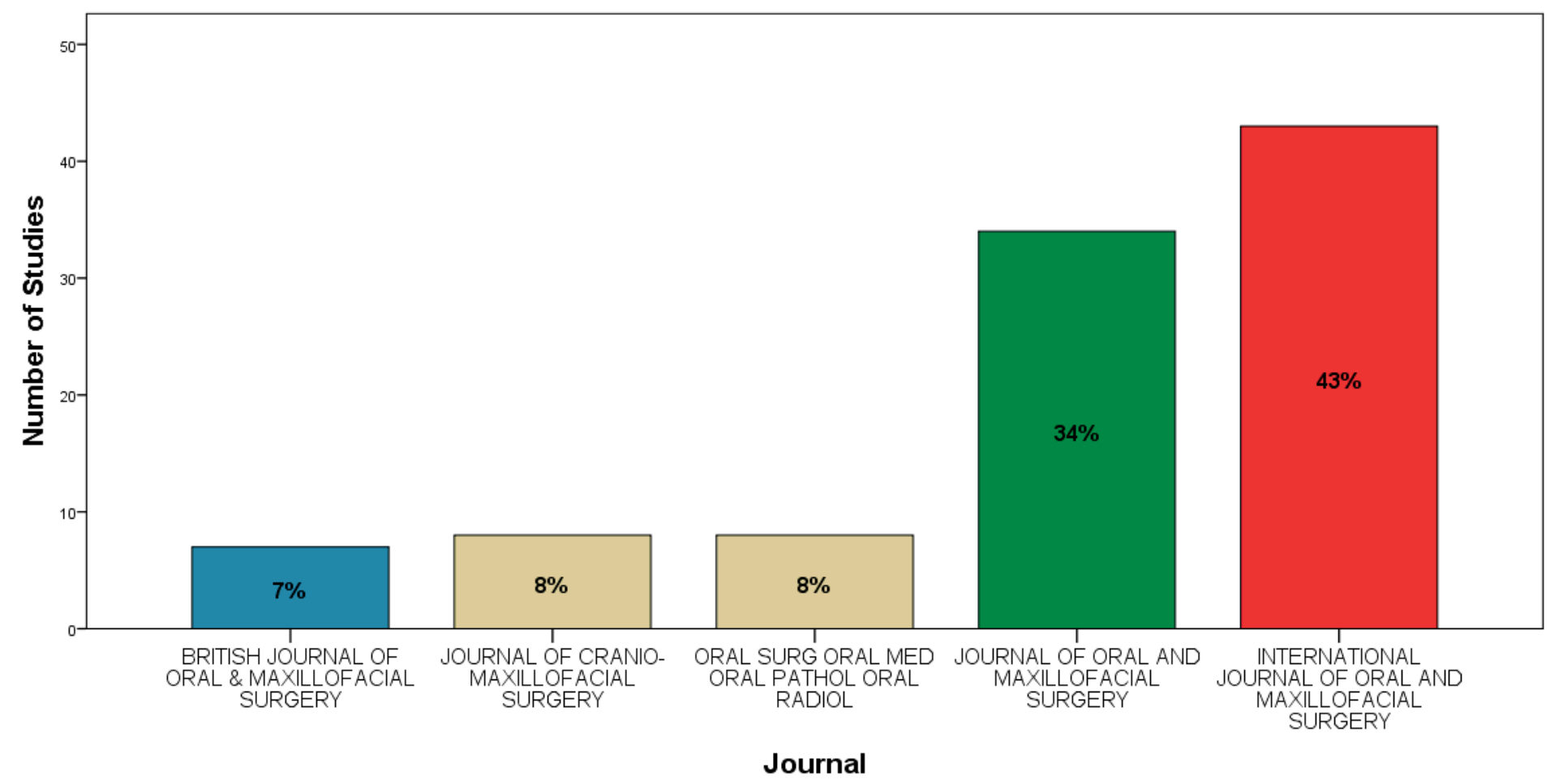

Figure 5

Most frequently cited journal

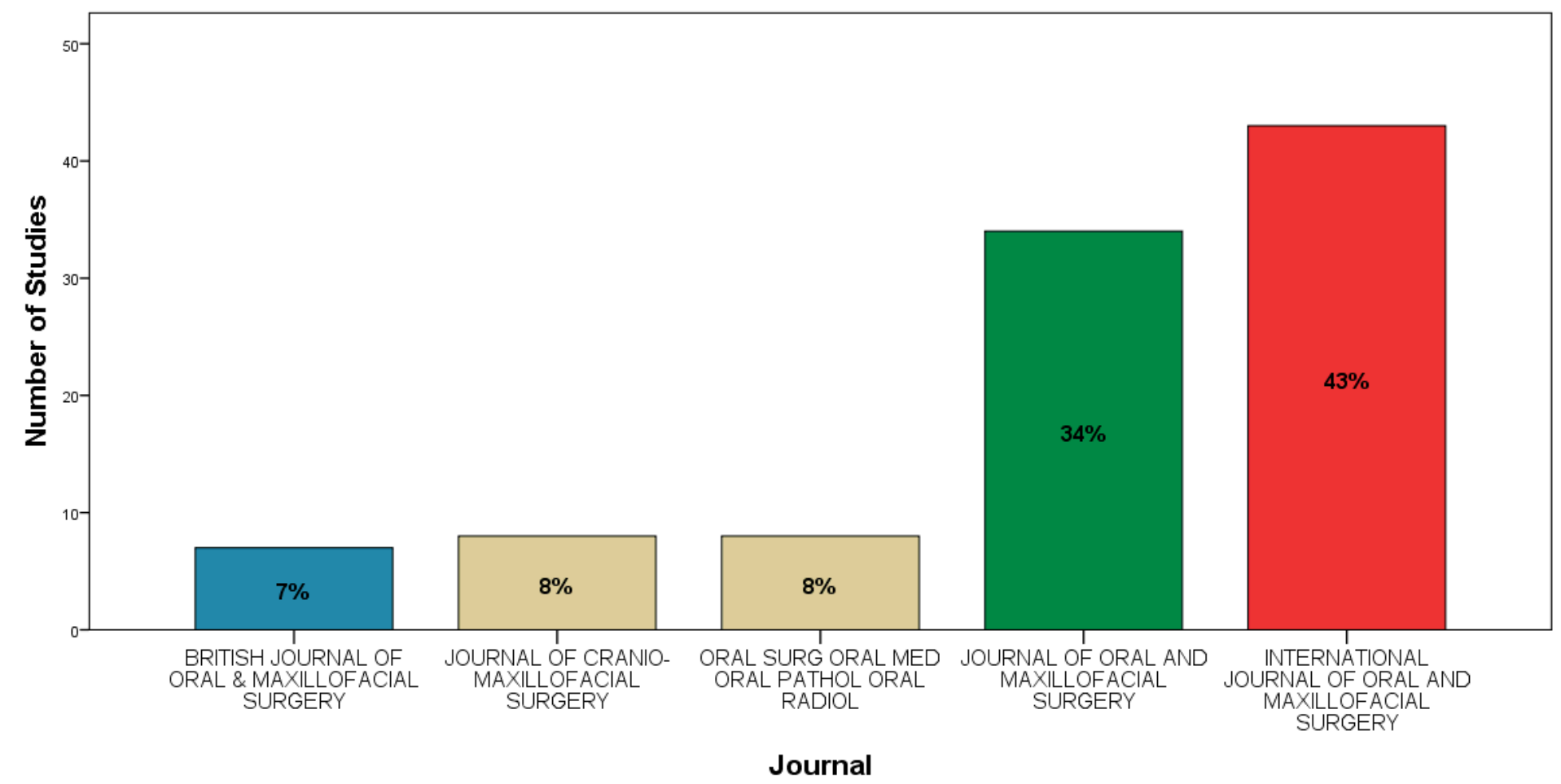

Figure 5

Most frequently cited journal 


\section{Supplementary Files}

This is a list of supplementary files associated with this preprint. Click to download.

- PRISMAPchecklist.docx

- PRISMAPchecklist.docx 